\title{
Large-Eddy Simulation of the Daytime Boundary Layer in an Idealized Valley Using the Weather Research and Forecasting Numerical Model
}

\author{
Franco Catalano · Chin-Hoh Moeng
}

Received: 15 October 2009 / Accepted: 7 June 2010 / Published online: 26 June 2010

(C) Springer Science+Business Media B.V. 2010

\begin{abstract}
A three-dimensional numerical meteorological model is used to perform largeeddy simulations of the upslope flow circulation over a periodic ridge-valley terrain. The subgrid-scale quantities are modelled using a prognostic turbulence kinetic energy (TKE) scheme, with a grid that has a constant horizontal resolution of $50 \mathrm{~m}$ and is stretched along the vertical direction. To account for the grid anisotropy, a modified subgrid length scale is used. To allow for the response of the surface fluxes to the valley-flow circulation, the soil surface temperature is imposed and the surface heat and momentum fluxes are computed based on Monin-Obukhov similarity theory. The model is designed with a symmetrical geometry using periodic boundary conditions in both the $x$ and $y$ directions. Two cases are simulated to study the influence of along-valley geostrophic wind forcing with different intensities. The presence of the orography introduces numerous complexities both in the mean properties of the flow and in the turbulent features, even for the idealized symmetric geometry. Classical definitions for the height of the planetary boundary layer (PBL) are revisited and redefined to capture the complex structure of the boundary layer. Analysis of first- and second-moment statistics, along with TKE budget, highlights the different structure of the PBL at different regions of the domain.
\end{abstract}

Keywords Boundary-layer height - Geostrophic wind forcing · Horizontal breeze . Subsidence $\cdot$ Surface flux heterogeneity $\cdot$ Turbulent kinetic energy budget

F. Catalano $(\square)$

Department of Hydraulics, Transportation and Roads, Sapienza University of Rome,

via Eudossiana 18, 00184 Rome, Italy

e-mail: franco.catalano@uniroma1.it

C.-H. Moeng

Mesoscale and Microscale Meteorology Division, National Center for Atmospheric Research (NCAR), 3450 Mitchell Lane, Boulder, CO, USA 


\section{Introduction}

Most severe events of atmospheric pollution occur under weak synoptic systems when the circulation is mainly driven by local forcing, and where mid-latitude zones with complex orography are mainly affected by slope and valley winds (Simpson 1994). Efforts are underway in developing wind farms, often located in mountainous zones; to generate efficient wind power-plants requires detailed and reliable knowledge of wind fluctuations near the surface that cannot be inferred from regional-scale investigations. Planetary boundary-layer (PBL) parameterization schemes in regional-scale models at current operational resolutions do not take into account the orographic effects on the vertical diffusion and in predicting wind fluctuations (Noppel and Fiedler 2002).

The basic physical mechanism of thermally-induced circulations in a valley is baroclinic instability driven by the daily cycle of solar radiation; horizontal pressure gradients formed by slope heating during the day lead to the generation of an upslope flow, while nocturnal cooling of the terrain induces a downslope gravity current. Field studies (Brehm and Freytag 1982; Kondo et al. 1989; Monti et al. 2002; Haiden and Whiteman 2005) have focused mainly on the description of the average features of circulations, such as flow depth and intensity; these campaigns have provided observations for evaluating and testing parameters used in analytical models. Theoretical models were developed for the upslope wind over a finite slope (Hunt et al. 2003) and for the downslope flow over an infinite slope (Manins and Sawford 1979); these models have been used to estimate bulk properties under simplified assumptions. The main features of upslope and downslope flows have been investigated at the laboratory scale using water tanks and image analysis techniques (e.g., Princevac and Fernando 2007). A drawback of this experimental approach is the inability to reproduce high Reynolds number flows with turbulence properties comparable to that of the real atmosphere. Numerical studies of the daytime PBL in a two- and a three-dimensional valley-plain system were performed on the fine mesoscale range (1- km horizontal resolution and approximately $50-\mathrm{m}$ vertical resolution) by Rampanelli et al. (2004) using the Weather Research and Forecast (WRF) model. Mesoscale modelling was also used to investigate the energy redistribution from local to regional scales and to test an analytical description of the valley circulation (Noppel and Fiedler 2002). These mesoscale modelling studies used one-dimensional PBL schemes that are crude representations of turbulence; they do not provide detailed turbulence structure and accurate statistics.

In the last three decades, owing to a progressive increase in computer power, large-eddy simulation (LES) has become an important tool for studying atmospheric turbulence. LES explicitly calculates large turbulent eddies that carry most of the turbulent kinetic energy and fluxes, while parameterizing the net effects of the small-scale component of turbulence. Hence LES provides a three-dimensional, time evolving structure of turbulence and a much more accurate estimate of turbulence statistics compared to those based on PBL schemes. Since the pioneering work of Deardorff (1972), a considerable and increasing effort has been made on the development and applications of LES to study atmospheric turbulence in the neutral and convective boundary layers (Moeng 1984; Nieuwstadt et al. 1993; Sullivan et al. 1994), and recently also to study the stably stratified boundary layer (SBL). The SBL is characterized with smaller eddies and requires a significantly finer resolution and more sophisticated subgrid-scale (SGS) schemes (Saiki et al. 2000; Beare et al. 2006). However, most largeeddy simulations were conducted assuming simple idealized boundary conditions, such as horizontal homogeneity with steady forcing or horizontal inhomogeneity with periodic wavy surfaces. Only a few large-eddy simulations were performed for the PBL over non-uniform terrain: Schumann (1990) investigated the diurnal cycle of the PBL over an infinite slope 
using a constant surface heat flux to examine the formation of transverse convective rolls associated with the upslope wind. Skyllingstad (2003) investigated the turbulent structure of a downslope wind over an infinite slope with constant cooling at the bottom. Chow et al. (2006) and Weigel et al. (2006) studied the PBL within the Riviera Valley (Switzerland), focusing mainly on the mean fields in the PBL. These studies also analyzed the heat budget but, due to their resolution of $150 \mathrm{~m}$ in the horizontal and $20 \mathrm{~m}$ in the vertical, their results showed the significance of the modelled subgrid-scale components of the turbulent fluxes compared to the resolved ones. Michioka and Chow (2008) used LES with a horizontal resolution of $25 \mathrm{~m}$ for the Mount Tsukuba (Japan) area but focused mainly on the dispersion characteristics of a passive scalar.

Our study aims at investigating both the mean quantities and turbulent variances and fluxes that characterize the daytime circulation over a valley with large-eddy simulation based on the WRF model. In order to reduce the degrees of freedom of the system and to isolate the interacting phenomena we limit the study to a simple periodic ridge-valley terrain. The model characteristics along with the simulation set-up are briefly discussed in Sect. 2. Section 3 presents the flow features and turbulence statistics that develop for the daytime convective PBL and the transition to the nighttime stable atmosphere. Section 4 examines the effect of the geostrophic wind along the valley, and Sect. 5 summaries the results.

\section{Model and Experiment Set-Up}

The three-dimensional meteorological model WRF is used as a framework for the LES simulations in the present work. WRF is a fully compressible, non-hydrostatic model with a terrain-following hydrostatic-pressure vertical coordinate. The model has been shown to be a good tool for LES (Moeng et al. 2007), but still needs to be thoroughly tested to perform LES in complex geometries. In a recent work, Lundquist et al. (2010) performed high resolution simulations of the flow over an urban skyline with WRF using the immersed boundary method but they did not present turbulent fluxes or perform a turbulence kinetic energy (TKE) budget analysis. Adopting the WRF model for the present work provides a step towards developing a multi-scale model for the real-world PBL that often consists of both mesoscale circulations and microscale turbulence.

Since the scales of the investigated phenomena are quite small we neglect the Coriolis terms in the LES equations, but implicitly include the Coriolis effect in the geostrophic forcing. We also limit our study to a dry atmosphere. For a detailed description of the WRF model and its basic equations we refer to Skamarock et al. (2008).

\subsection{The SGS Model}

For LES applications, we turn off PBL schemes and use a subgrid-scale model to represent the small-scale component of turbulent motions. The SGS model used here is based on the WRF TKE diffusion scheme proposed by Deardorff (1980) but with some modifications to take into account the effects of our highly anisotropic grid. Indeed, according to Sagaut (2006), with an aspect ratio $\Delta z / \Delta x$ of about eight subgrid stresses can differ by about $10 \%$ with respect to those computed on an isotropic grid for isotropic and homogeneous turbulence. With inhomogeneous and anisotropic turbulence the impact of anisotropic grids may be less dramatic but still significant. The turbulent viscosity and diffusivity $K_{m}$ and $K_{h}$ are expressed as: 


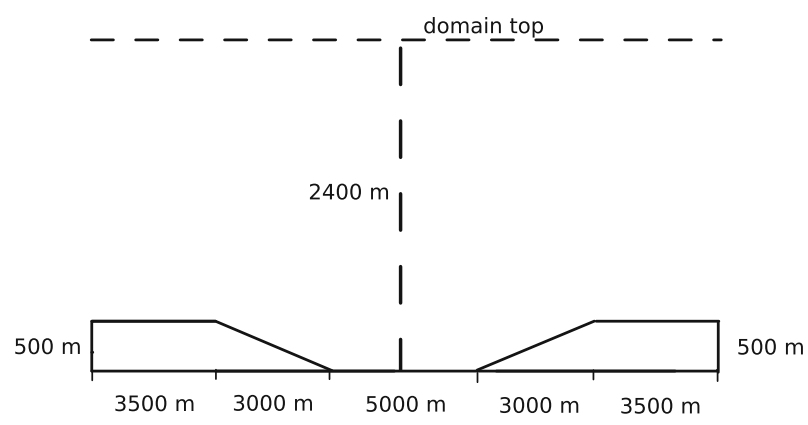

Fig. 1 Vertical view of domain geometry. The vertical axis has been exaggerated for a better visualization; the width along the $y$ axis is $5,000 \mathrm{~m}$

$$
\begin{aligned}
K_{m} & =C_{k} l \sqrt{e}, \\
K_{h} & =K_{m} \frac{1}{P r_{t}},
\end{aligned}
$$

where $C_{k}=0.15$ is the diffusion coefficient, $e$ is the SGS TKE computed from an additional prognostic equation (Moeng 1984), $1 / \operatorname{Pr}_{t}=1+2 l / \Lambda$ is the inverse turbulent Prandtl number, and $\Lambda$ is the LES filter width. The SGS length scale $l$ is defined as:

$$
l= \begin{cases}\min \left[\Lambda, 0.76 \frac{\sqrt{e}}{N}\right] & \text { for } N^{2}>0 \\ \Lambda & \text { for } N^{2} \leq 0\end{cases}
$$

where $N^{2}=(g / \theta) \partial \theta / \partial z$ is the square of the Brunt-Vaisälä frequency, $g$ is the gravity acceleration and $\theta$ is the potential temperature. A common definition of the LES filter width $\Lambda$ is the geometrical mean of the dimensions of the cell $\Delta=(\Delta x \Delta y \Delta z)^{1 / 3}$ (Deardorff 1970), which is appropriate only for nearly isotropic grids. In this study, we use a vertically stretched grid to obtain a finer vertical grid resolution near the surface, which leads to a very small aspect ratio $\Delta z /(\Delta x, \Delta y) \ll 1$. Scotti et al. (1993) demonstrated that for grids with a small aspect ratio the common approach causes an underestimation of the turbulent viscosity $K_{m}$ and suggested the following modification using a correction function $f\left(a_{1}, a_{2}\right)$ :

$$
\begin{gathered}
\Lambda=f \Delta, \\
f=\cosh \sqrt{\frac{4}{27}\left[\left(\ln a_{1}\right)^{2}-\left(\ln a_{1}\right)\left(\ln a_{2}\right)+\left(\ln a_{2}\right)^{2}\right]}, \\
a_{1}=\frac{\Delta_{i}}{\max [\Delta x, \Delta y, \Delta z]}, \\
a_{2}=\frac{\Delta_{k}}{\max [\Delta x, \Delta y, \Delta z]},
\end{gathered}
$$

where $a_{1}$ and $a_{2}$ are the aspect ratios of the two other sides of the grid relative to the longest side and $\Delta_{i}$ and $\Delta_{k}$ are the lengths of those two sides. For example, if $\max [\Delta x, \Delta y, \Delta z]=\Delta z$, then $a_{1}=\Delta x / \Delta z$ and $a_{2}=\Delta y / \Delta z$. This formulation results in an increase of the filter width (and hence the eddy viscosity) in the zones of the domain where the aspect ratios deviate away from one. The modified WRF TKE diffusion scheme is used to model the effects of the SGS turbulence. 


\subsection{The Numerical Set-Up}

The domain geometry shown in Fig. 1 consists of a valley that is uniform in $y$ and symmetric in $x$ about the centre of the valley (i.e., at $x=L_{x} / 2$ where $L_{x}$ is the length of the domain in $x$ ). The valley has two lateral ridges on both sides, and the slopes have a steep inclination of $\approx 16 \%$ and cover $3,000 \mathrm{~m}$ distance in $x$. The width of the domain along the $y$ axis is $5,000 \mathrm{~m}$. The horizontal grid size is $\Delta x=\Delta y=50 \mathrm{~m}$, while the vertical grid is stretched with $\Delta z$ defined by a parabolic function of $z$ and varies from $\approx 2 \mathrm{~m}$ near the bottom of the valley to $\approx 90 \mathrm{~m}$ at the top of the domain. Since the vertical coordinate is terrain following and the top of the domain is fixed, the vertical resolution slightly increases with ground elevation. For a constant and homogeneous horizontal grid we have:

$$
\begin{aligned}
& a_{1}=\frac{\Delta z}{\Delta x}, \\
& a_{2}=1,
\end{aligned}
$$

for $\Delta z<\Delta x$,

$$
a_{1}=a_{2}=\frac{\Delta x}{\Delta z}
$$

for $\Delta z>\Delta x$.

Hence there is a moderate increase of the filter width near the ground $(\Delta z<\Delta x)$ and a slight increase close to the top of the domain $(\Delta z>\Delta x)$. We use a fifth-order advection scheme for horizontal advection terms, a third-order scheme for vertical advection and a third-order Runge-Kutta (RK) scheme for the time integration with a timestep $\Delta t=1 \mathrm{~s}$. We chose $\Delta t / \Delta t_{s}=12$, where $\Delta t_{s}$ is a small timestep for the integration of acoustic wave equations. The WRF code contains optimized algorithms for the damping of acoustic wave modes; here we set the three-dimensional divergence damping coefficient to 0.1 , the external wave filter coefficient to 0.01 , and the small acoustic timestep off-centering coefficient to 0.1 .

Periodic lateral boundary conditions are imposed in $x$ and $y$, so the valley flow and turbulence statistics are symmetric about $x=L_{x} / 2$. The top boundary of the domain is assumed to be at constant pressure with zero vertical velocity. A preliminary analysis (not shown) suggested a negligible influence of the reflections of gravity waves on the solution and hence an upper damping layer is not needed. The choice is supported by previous mesoscale (Rampanelli et al. 2004) and LES (Moeng et al. 2007; Antonelli and Rotunno 2007) studies of the atmospheric boundary layer conducted with the WRF model. The lower boundary condition is described by a sinusoidal thermal forcing:

$$
\Delta \bar{\theta}_{s}=\Delta \bar{\theta}_{s, \max } \sin \left[\frac{2 \pi t}{T}\right],
$$

where $\Delta \bar{\theta}_{s}$ is the increment of the surface temperature with time (defined with respect to the values at $t=0), \Delta \bar{\theta}_{s, \max }=5 \mathrm{~K}$ is the amplitude, $T=24 \mathrm{~h}$ is the period, and $t$ is the time. The initial sounding has a horizontally uniform lapse rate of $N^{2}=1.6 \times 10^{-4} \mathrm{~s}^{-2}$ and surface temperature in the valley as $\bar{\theta}_{S}(z=0, t=0)=300 \mathrm{~K}$; the initial soil temperature is then a function of the height of the ground relative to the bottom of the valley $\left(z_{s}\right)$. The surface heat flux and the friction velocity are computed according to Monin-Obukhov similarity theory. We neglect the effects of the variations of the solar radiation direction, the shadowing from the surrounding terrain and the influence of the deviation from the zenith direction. The terms west-east and south-north refer, respectively, to the orientation of $x$ and $y$ axes. Due to the above mentioned assumptions, this is an idealized valley flow simulation. 


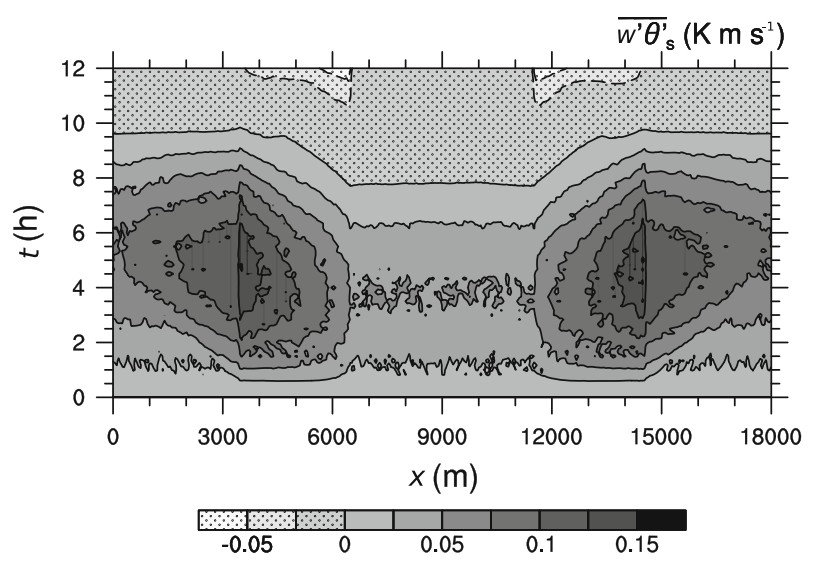

Fig. 2 Hovmoller plot of the $y$-averaged surface kinematic heat flux for Case $1\left(V_{g}=2 \mathrm{~m} \mathrm{~s}^{-1}\right)$

Two cases are investigated to study the influence of along-valley geostrophic wind $V_{g}$ on the thermal circulation and turbulence properties. Case 1 with $V_{g}=2 \mathrm{~m} \mathrm{~s}^{-1}$ represents a situation that is likely to occur in a real PBL for weak synoptic systems, while Case 2 is driven by a larger geostrophic wind of $V_{g}=10 \mathrm{~m} \mathrm{~s}^{-1}$. In both cases the symmetry in $x$ is preserved by setting the cross-valley component of the geostrophic wind $U_{g}=0$. The surface roughness length $z_{0}$ is set to $0.3 \mathrm{~m}$, which is typical of rough land (brush).

The total grid points are 360 in $x, 100$ in $y$, and 58 in $z$, and the model is integrated for $12 \mathrm{~h}$, starting from sunrise, to simulate the daytime PBL cycle. A preliminary investigation revealed that our grid resolution is not fine enough to fully resolve the turbulent eddies in the nighttime PBL (Saiki et al. 2000); hence we cease the simulation immediately after the transition. Small random perturbations (with a maximum amplitude of $0.05 \mathrm{~K}$ ) are applied to the initial temperature at the first four model layers and at the surface. We have verified (not shown) that after an initial spin-up time of $30 \mathrm{~min}$ the flow can be considered fully turbulent, in agreement with the LES results of Nieuwstadt et al. (1993) for free convection.

\section{Results}

Since the valley flow and the PBL turbulence are driven mainly by surface heating, we first present the time evolution of the $y$-averaged surface heat flux in Fig. 2, for Case 1. In the valley, the surface heat flux reaches its maximum around $4 \mathrm{~h}$ even though the ground temperature is greatest around $6 \mathrm{~h}$. This shows evidence of strong coupling of the surface to the valley flow, which results in the largest difference between the surface and the near-surface air temperature at $4 \mathrm{~h}$. Over the ridge, the maximum heat flux occurs around $5 \mathrm{~h}$. The sharp peak near the top of the slope is about twice the maximum value over the valley. The time cycle over the slope region shows an intermediate situation between the ridge and the valley, with a greater flux close to the ridge. The surface flux becomes negative after $\approx 8 \mathrm{~h}$ over the valley and after $\approx 9.5 \mathrm{~h}$ over the ridge, the largest negative heat flux occurs at $t \approx 12 \mathrm{~h}$ near the foot of the slope. This complex spatial and temporal evolution of the surface heat flux is typical of steep alpine valleys, as documented in the field measurements of Rotach et al. (2004) and the numerical simulations of Chow et al. (2006), and can be reproduced here only through the computation of the surface heat fluxes from a coupled surface-layer scheme. 

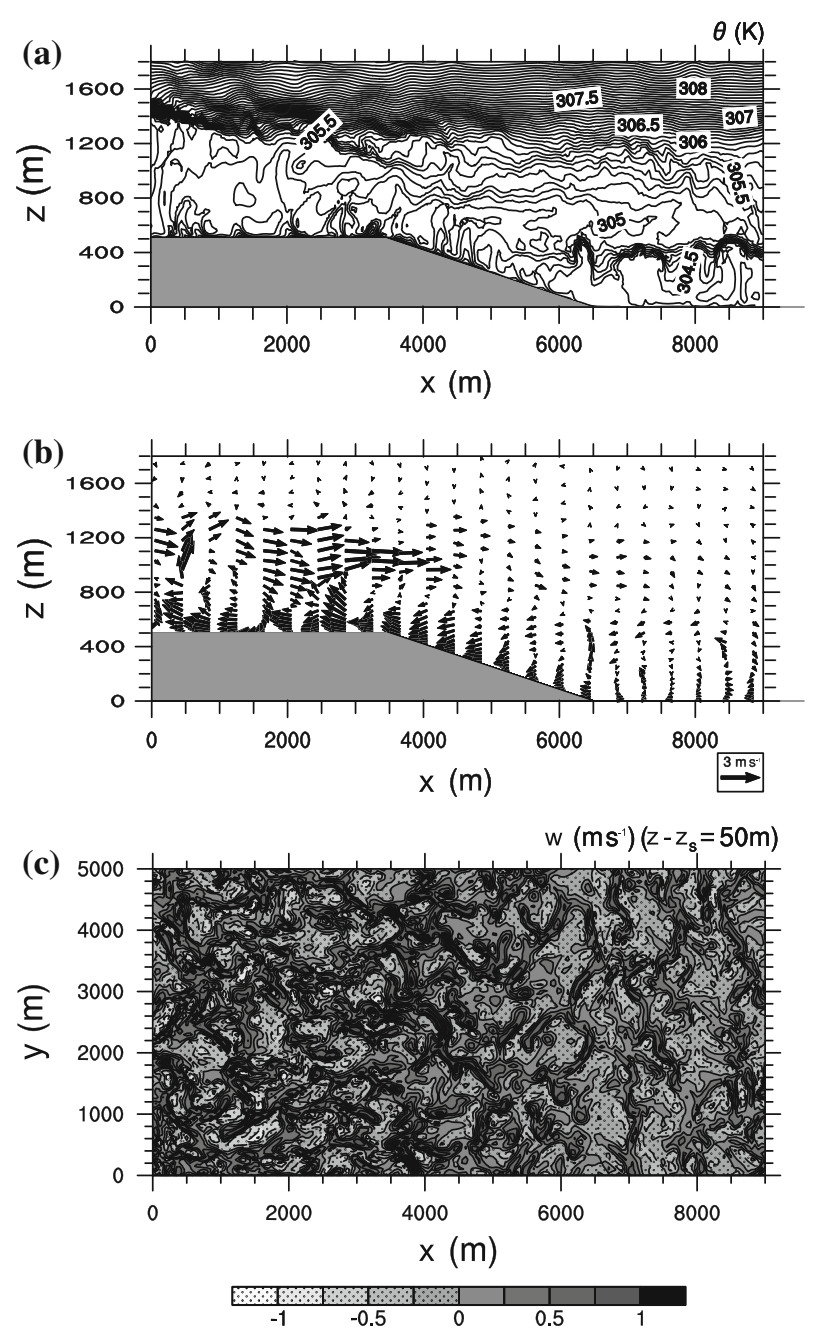

Fig. 3 Instantaneous fields at $t=7 \mathrm{~h}$ for Case $1\left(V_{g}=2 \mathrm{~m} \mathrm{~s}^{-1}\right)$ : vertical cross-sections of isotherms (a) and vectors (b) at $y=L_{y} / 2$; horizontal cross-section of the vertical velocity (c) at a fixed relative height from the ground

\subsection{Instantaneous Flow Fields}

Due to the symmetrical geometry of the numerical domain, the simulated flow field remains symmetrical about the vertical plane at $x=L_{x} / 2$. Henceforth, in most contour plots we show just the western half of the domain. One of the most important advantages of the LES approach is its ability to resolve the turbulent structures of the flow. The instantaneous flow field at $t=7 \mathrm{~h}$ of the simulation, when the daytime circulation is well developed, displays both terrain-induced mesoscale circulations as well as the random turbulent motions. The potential temperature field (Fig. 3a) shows a shallow mixed layer with a well-defined inversion at $z \approx 400 \mathrm{~m}$ over the valley and a deep mixed layer with an inversion at $z \approx 1,300$ $\mathrm{m}$ over the ridge, while the wind field (Fig. $3 \mathrm{~b}$ ) reveals a large circulation with an upslope 
flow near the surface and a return flow below $z \approx 1,300 \mathrm{~m}$. The streamline also suggests a secondary circulation (i.e., clockwise between $z \approx 400$ and $1,300 \mathrm{~m}$ ) in the upper part of the valley and slope regions, which will be described later.

Figure $3 \mathrm{c}$ provides a horizontal view of vertical velocity fluctuations at $50 \mathrm{~m}$ above the ground. An irregular cellular pattern is observed over the ridge and slope areas, which is typical of a convective boundary layer (CBL); the turbulent fluctuations are much weaker in the valley. As shown, the surface heat flux at this simulation hour is positive at all locations but it is at least three times smaller over the valley. The horizontal temperature gradient over the slope produces an upslope flow near the surface from the valley towards the ridges; this is also reflected in the observed surface heat-flux differences in the different regions of the domain. Over the valley the eddy structure is oriented along the $y$ direction; this elongated feature is related to the vertical shear induced by the geostrophic wind forcing and its interaction with the surface buoyancy.

\subsection{Averaging Procedures for Computing Statistics}

Most previous LES studies investigated the idealized PBL over horizontal homogeneous surfaces (both in $x$ and $y$ directions), so the averaging procedure in defining turbulence statistics was straightforwardly obtained by horizontal averaging. This approach guaranteed a sufficient number of sample points for the robustness of the statistics, even with domains of modest sizes. In the present case the only statistically homogeneous direction is along the $y$-axis, and so the perturbations $\phi^{\prime}$ are defined:

$$
\phi^{\prime}(x, y, z, t)=\phi(x, y, z, t)-\bar{\phi}(x, z, t),
$$

where $\phi$ is the instantaneous value of the considered variable,

$$
\bar{\phi}=\frac{1}{L_{y}} \sum_{j=1}^{N_{y}} \phi_{j}
$$

is its average value along the $y$ direction, and $L_{y}$ is the length of the domain in the $y$ direction. The averaging length $L_{y}$ has to be more than ten times the turbulence integral scale in order to obtain reliable statistics (Wyngaard 1983). In the present study, we set $L_{y}=5 \mathrm{~km}$, which is a compromise between the needs for a long averaging length and the computational demand. To improve the data sample for reliable statistics, all moment statistics are subsequently averaged in time over a period of $40 \mathrm{~min}$.

\subsection{Distributions of First-Moment Statistics}

The averaged potential temperature at $7 \mathrm{~h}$ in Fig. 4a shows a deep well-mixed PBL in the ridge region where the PBL is capped by a well-defined inversion layer above $z \approx 1,300 \mathrm{~m}$. In the valley, the PBL is much shallower with a capping inversion between $z=300$ and $450 \mathrm{~m}$. Below the free atmosphere $(z<1,200 \mathrm{~m})$ two distinct turbulent layers can be identified over the valley $(6,500 \mathrm{~m}<x<11,500 \mathrm{~m})$ at this time, separated by the thermal inversion: the lower one is quite uniform and extends up to $z \approx 400 \mathrm{~m}$, while the upper one is located at $500 \mathrm{~m}<z<1,200 \mathrm{~m}$ and is horizontally inhomogeneous. The inversion is maintained by (a) warm-air advection from the return flow, and (b) subsidence warming due to the sinking motion of the induced circulation. A relevant effect of this twofold warming mechanism is the suppression of the development of the CBL in the basin, as confirmed by the measurements of Kondo et al. (1989). The mean temperature field shows a horizontal gradient of the 

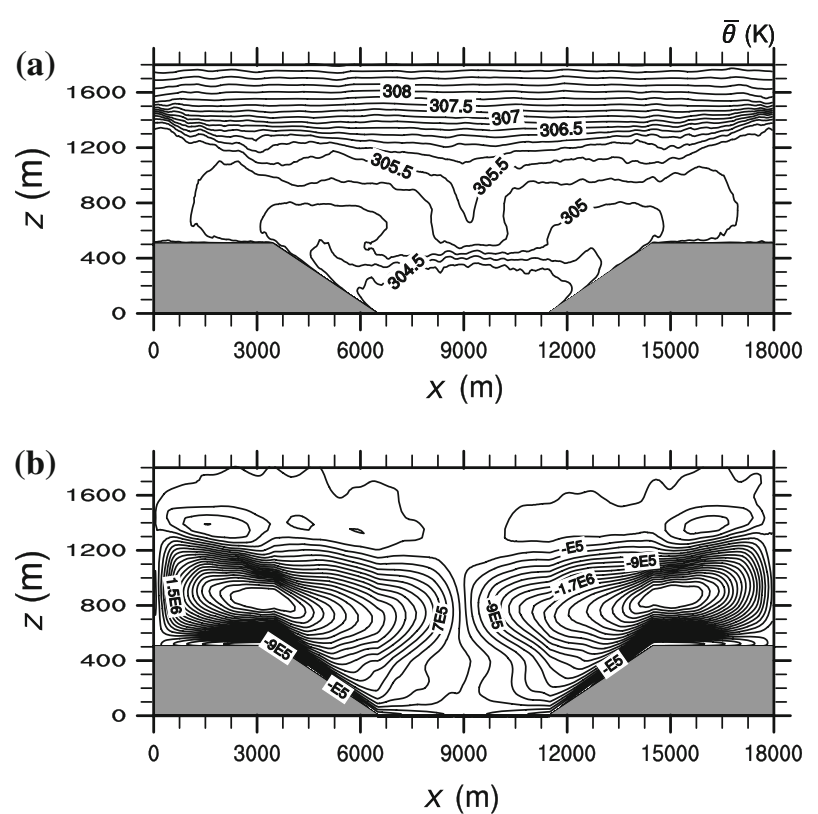

Fig. $4 y$-averaged vertical cross-sections of isotherms (a) and stream function (b) at $t=7 \mathrm{~h}$ for Case 1 $\left(V_{g}=2 \mathrm{~m} \mathrm{~s}^{-1}\right)$

near-surface temperature along $x$, which leads to the development of an upslope flow over the two slopes as revealed by the streamfunction of the averaged wind fields (Fig. 4b).

Figure 5 shows the three components of the mean wind at $7 \mathrm{~h}$ : the maximum intensity of the upslope wind is attained at $t=5 \mathrm{~h}$ of simulation (not shown). After that time, the depth of the upslope flow continues to rise and eventually merges with a horizontal breeze (westward) located between $z=400$ and $800 \mathrm{~m}$ and extending from the valley to the slope region (Fig. 5a). This horizontal breeze occurs at the terrain-height level of the ridge and is driven by the horizontal pressure gradient between the valley and the ridge. The horizontal breeze, along with the return flow, induces a secondary circulation in the upper part $(400 \mathrm{~m}<z<1,300 \mathrm{~m}$ ) of the domain. This secondary circulation may affect the air quality of cities in such an environment enhancing the dispersion of pollutants into the upper air (above the mixed layer) over the valley. This upper-level circulation is maintained by the subsidence over the centre of the valley and the presence of a strong inversion at $z \approx 400 \mathrm{~m}$ over the valley. The inversion forces the downward flow to diverge and move horizontally towards the ridges. Note that between $z \approx 500 \mathrm{~m}$ and $z \approx 800 \mathrm{~m}$ the potential temperature of the air above the slope is lower than that over the valley (Fig. 4), and hence the horizontal breeze moves against the horizontal gradient of temperature. The horizontal breeze was not observed in previous investigations of the valley circulation, and might be due to the more intense synoptic conditions. In fact the field measurements of Rotach et al. (2004) and the large-eddy simulations of Chow et al. (2006) show an upper level cross-valley wind speed of $\approx 15 \mathrm{~m} \mathrm{~s}^{-1}$.

The along-valley component of the wind (Fig. 5b), forced by the prescribed geostrophic wind, shows a tunnelling effect in the centre of the valley, where a shallow layer with wind speeds of about $1.8 \mathrm{~m} \mathrm{~s}^{-1}$ is present between 400 and $500 \mathrm{~m}$. This layer coincides with the westward horizontal breeze layer seen in Fig. 5a, together suggesting a north-westward 

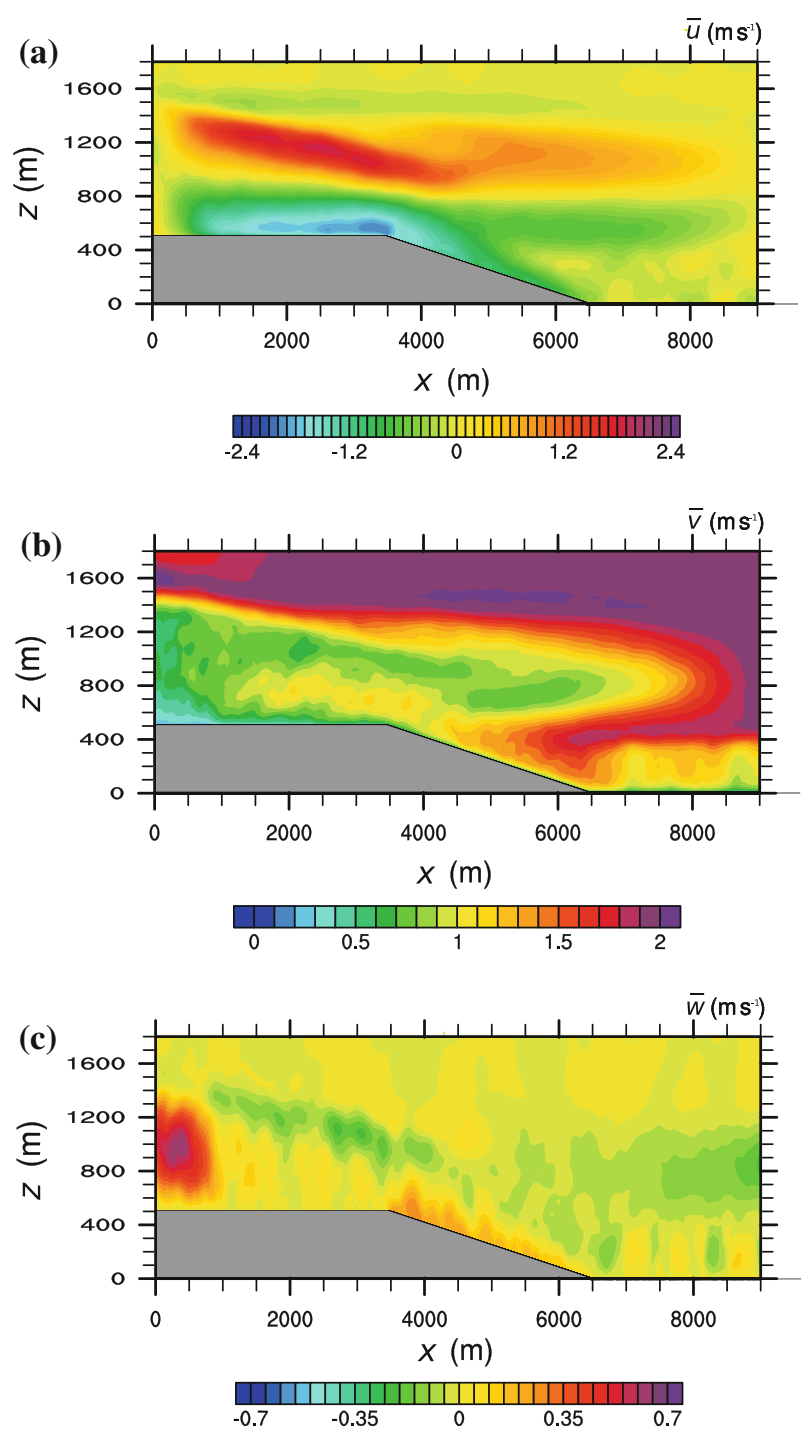

Fig. $5 y$-averaged vertical cross-sections of the wind components: $\bar{u}(\mathbf{a}), \bar{v}(\mathbf{b}), \bar{w}(\mathbf{c})$ at $t=7 \mathrm{~h}$ for Case 1 $\left(V_{g}=2 \mathrm{~m} \mathrm{~s}^{-1}\right)$

current confined to the top of the PBL in the valley, and this current extends to the slope region and lies between the upslope flow and the return current.

The vertical velocity (Fig. 5c) shows strong updrafts around $x=0$ where the two upslope flows converge (due to the periodic boundary condition in $x$ ), with the characteristic depth of the mean updrafts decreasing towards the valley as the surface heat flux decreases. A broad mean subsidence extending over the whole valley can be observed between $z \approx 500 \mathrm{~m}$ and $z \approx 1,100 \mathrm{~m}$ with subsidence velocities of $\approx 0.1 \mathrm{~m} \mathrm{~s}^{-1}$. Similar large subsidence values have been observed by Hennemuth (1987) for the Dischma valley (Switzerland) and are in agreement with the numerical results of Rampanelli et al. (2004). 
Fig. 6 Profiles of the $y$-averaged potential temperature $\bar{\theta}$ at

different $x$ locations at $t=7 \mathrm{~h}$ for Case $1\left(V_{g}=2 \mathrm{~m} \mathrm{~s}^{-1}\right)$ as a function of the non-dimensional vertical coordinate $z_{r} / z_{i}=$ $\left(z-z_{s}\right) / z_{i}$, where $z_{i}$ is the PBL depth and $z_{s}$ is the local terrain elevation. Filled circles refer to the valley centre, open squares refer to the middle of the left slope and filled triangles refer to the middle of the left ridge

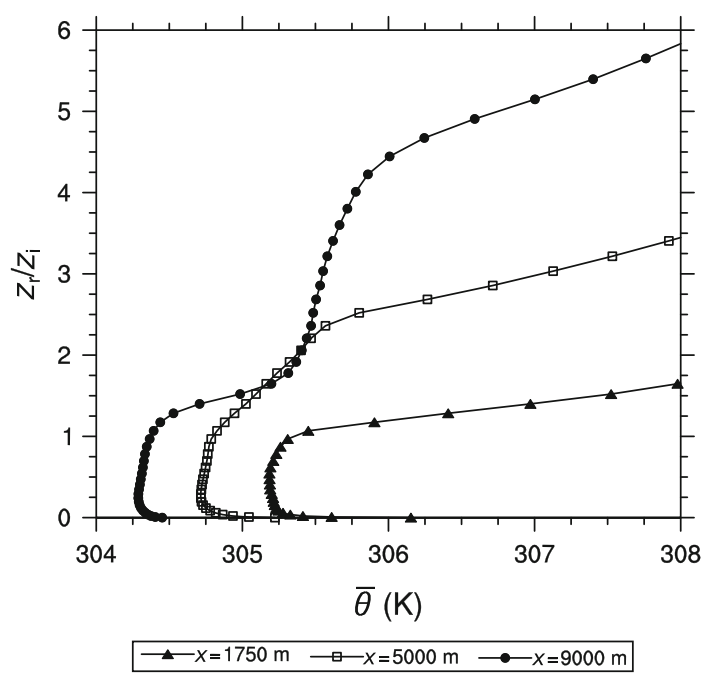

\subsection{The PBL Depth}

Due to the variable orography, the height of the PBL $z_{i}$ must be defined locally and relative to its ground level $z_{s}$. Also, different definitions must be given depending on the stability regime, which varies with time and $x$ location, as seen from the sign of the surface heat flux (Fig. 2). The complex and spatially varying PBL structure makes the classical definitions for the convective boundary layer (based on the minimum of the vertical heat flux or the maximum potential temperature gradient, Sullivan et al. 1998) inapplicable.

For the unstable regime $\left(\overline{w^{\prime} \theta^{\prime}}{ }_{s}>0\right)$ we propose to define $z_{i}$ as the height where the magnitude of the vertical gradient of potential temperature is greater than a critical value $\Gamma_{c}=0.001 \mathrm{~K} \mathrm{~m}^{-1}$ (i.e., at the base of a capping inversion) with the additional constraint that the heat flux is less than $15 \%$ of its maximum value. This definition is justified by the shape of the vertical profiles of the mean potential temperature at different $x$ locations at $t=7 \mathrm{~h}$ (Fig. 6), where the height relative to the ground $z_{r}=z-z_{s}$ has been normalized by $z_{i}$. While over the ridge there is a clear signal for a single inversion layer, over the slope and the valley two inversions exist; over the valley the lower inversion corresponds clearly to a sharp capping inversion, while this is not the case over the slope region. Because we exclude the entire capping inversion zone to be part of the PBL, our method may underestimate $z_{i}$ compared with the common definitions of the CBL depth.

For the near-neutral to moderately stable regimes $\overline{\left(w^{\prime} \theta^{\prime}\right.}{ }_{s} \leq 0$, e.g., during the evening transition) we distinguish two situations: (a) when the PBL structure is dominated by the surface wind shear (due to geostrophic forcing); and (b) when the effect of the temperature stratification (i.e., surface inversion) prevails. The friction velocity $u_{*}$ is used to distinguish the two conditions with an empirical threshold value of $0.2 \mathrm{~m} \mathrm{~s}^{-1}$. For the former case, $z_{i}$ is defined as the height where the along- $y$ component of wind shear $|\partial \bar{v} / \partial z|$ falls below a critical value $S_{c}=0.005 \mathrm{~s}^{-1}$, which is the height at which $v \approx V_{g}$. For $u_{*}<0.2 \mathrm{~m} \mathrm{~s}^{-1}$, the inversion strength approach proposed by Hyun et al. (2005) is applied and $z_{i}$ is defined at the level where the surface inversion strength $\delta=\theta-\bar{\theta}_{s}$ is smaller than $20 \%$ of its maximum value. We have also tested a method based on the values of TKE (not shown) that could be 


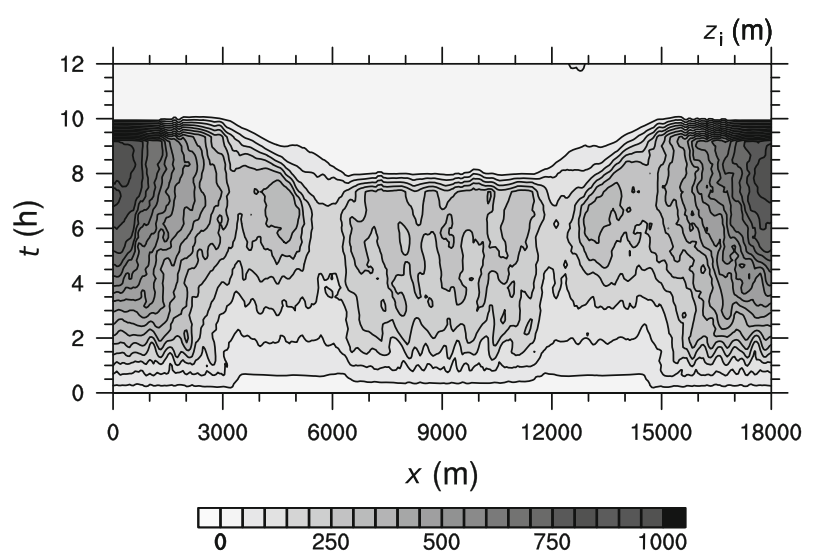

Fig. 7 Hovmoller plot of the $y$-averaged PBL depth $z_{i}$ for Case $1\left(V_{g}=2 \mathrm{~m} \mathrm{~s}^{-1}\right)$

applied to both unstable and stable regimes, but the presence of upper-level turbulence (to be discussed in the next sub-section) induced by valley flows makes this method impractical.

The time evolution of the PBL depth for Case 1 is shown in Fig. 7. We computed $z_{i}$ at each $(x, y)$ location and averaged along $y$ and over a time period of $40 \mathrm{~min}$. The plot shows a substantial increase of $z_{i}$ up to the time where the surface heat flux becomes negative (Fig. 2), and then the PBL depth falls abruptly to below $50 \mathrm{~m}$. As we will see in Sect. 4, this can be attributed to the weak geostrophic forcing that does not produce sufficient shear to generate turbulence against the negative surface heat flux. Maximum PBL depths of about $850 \mathrm{~m}$ are observed at both ends of the ridges in correspondence with the strong updrafts. The average PBL depth over the slope (about $400 \mathrm{~m}$ at its middle) is about $100 \mathrm{~m}$ higher than that over the valley, although this value does not consider the upper mixed layer that forms over the valley (see Fig. 6).

\subsection{Distributions of Second-Moment Statistics}

Figure 8a shows a vertical cross-section of the total TKE, along with the wind vectors at $7 \mathrm{~h}$; the black line shows the estimated PBL depth. At this time, the inclination of the growth line of $z_{i}$ over the ridge is close to the angle of the slope. The horizontal breeze at $z \approx 400 \mathrm{~m}$ over the slope does not produce a noticeable increase in the turbulence kinetic energy. Over the valley region, $z_{i}$ is about $300 \mathrm{~m}$ while the TKE remains significant at about $400 \mathrm{~m}$. Hence the method we proposed to compute $z_{i}$ underestimates the turbulence layer by about $25 \%$ over the valley in the presence of a strong capping inversion. To understand the source of TKE, we examined the individual components of the velocity variances. The SGS contributions to these variances are assumed to be $2 / 3$ of the predicted SGS TKE. Over the ridge, the most important contribution to the TKE is from the $u$ variance (Fig. 8b), except near the west boundary where $w$ variance dominates (Fig. 8d). The large contribution from the $u$ variance in the CBL over the ridge is unexpected, since the TKE in the bulk of a typical CBL is usually dominated by the $w$ variance instead (e.g., Moeng and Sullivan 1994). The dominance of the $u$ variance in TKE implies a significant shear production due to valley-flow circulation, which will be confirmed later. The turbulence over the slope is mostly determined by the $w$ variance, while the $v$ variance contribution (Fig. 8c) is relatively small, except in the inversion layer near the west-end boundary where the buoyancy flux is negative (Fig. 8e). Figure 8a and $\mathrm{b}$ also reveal double peaks in the $u$ variance profiles over the ridge and the upper part 

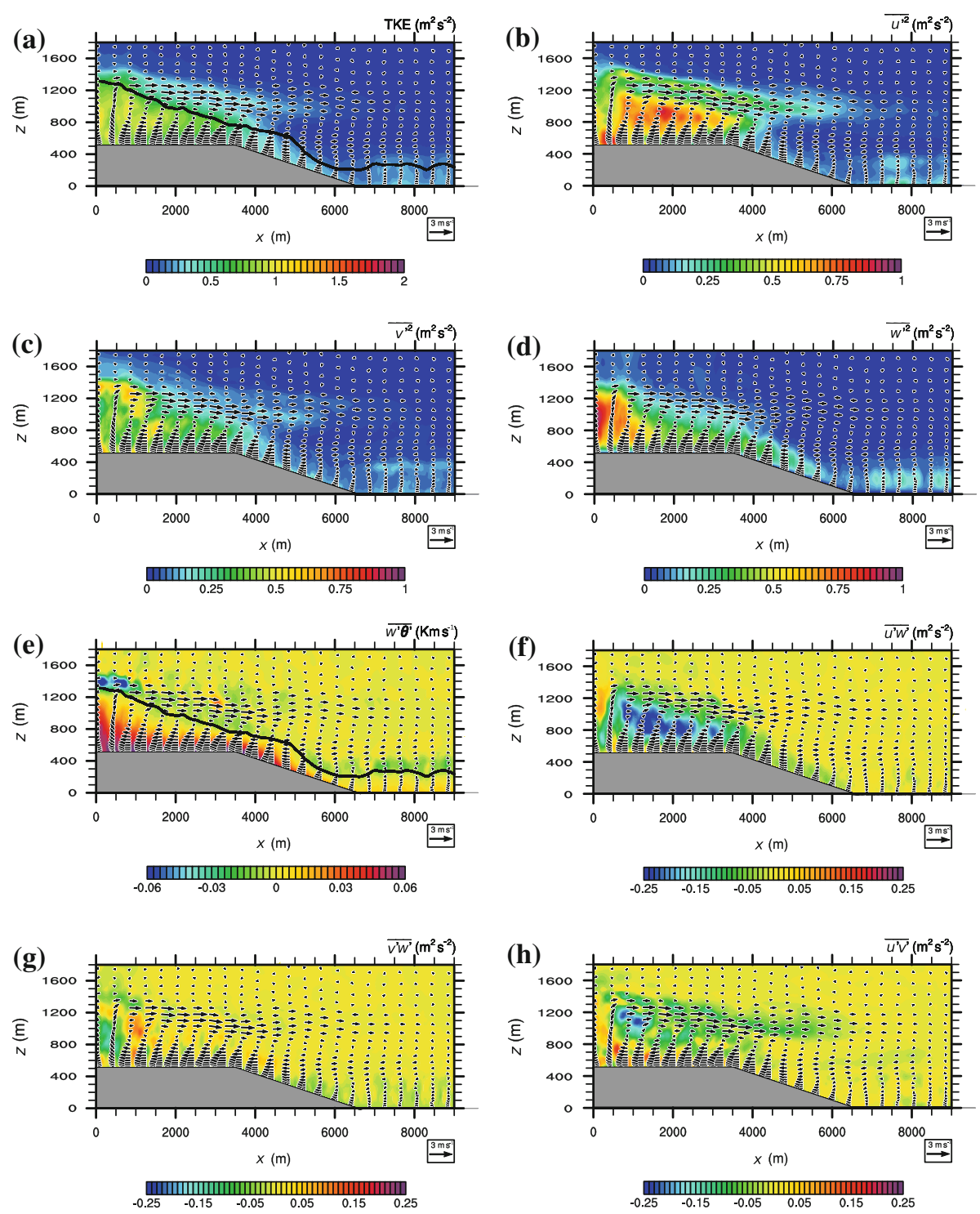

Fig. 8 Vertical cross-sections of the $y$-averaged second-moment statistics, along with the wind vectors: TKE (a), $\overline{u^{\prime 2}}(\mathbf{b}), \overline{v^{\prime 2}}$ (c), $\overline{w^{\prime 2}}(\mathbf{d}), \overline{w^{\prime} \theta^{\prime}}(\mathbf{e}), \overline{u^{\prime} w^{\prime}}(\mathbf{f}), \overline{v^{\prime} w^{\prime}}(\mathbf{g}), \overline{u^{\prime} v^{\prime}}(\mathbf{h})$ at $t=7 \mathrm{~h}$ for Case $1\left(V_{g}=2 \mathrm{~m} \mathrm{~s}^{-1}\right)$. The black line on panels (a) and (b) represents the $y$-averaged PBL depth

of the slope: one in the middle of the PBL and the other near the PBL top. The TKE budget shown later suggests that the upper maximum is due to the horizontal advection of the TKE. Overall the TKE over the ridge is about twice that over the slope and three times higher than that over the valley.

The vertical fluxes (resolved plus SGS) are shown in Fig. 8e-h. The heat flux is mostly positive except near the PBL top, which is typical of the CBL, while negative fluxes at the CBL top lie in the capping inversion regions, which agree well with our estimated $z_{i}$ shown in the same plot (Fig. 8e). The strong negative values of $\overline{u^{\prime} w^{\prime}}$ (Fig. 8f) and the significant 
positive gradients of mean $u$ over the ridge (Fig. 5a) yield a large shear production for TKE, which explains the large contribution of $u$ variance to the TKE in that region. In the valley, negative $\overline{v^{\prime} w^{\prime}}$ (Fig. 8g) coincides with a positive $v$ gradient in the lower PBL (Fig. 5b), which also contributes to shear production of TKE. The horizontal flux $\overline{u^{\prime} v^{\prime}}$ shown in Fig. $8 \mathrm{~h}$ will be used to discuss the TKE budget later.

In our simulations we reproduce the interaction of three forcing mechanisms: (a) buoyancy resulting from the surface heat flux; (b) baroclinic shear induced by the valley-flow circulation; (c) shear generated by the imposed geostrophic forcing. Even if buoyancy dominates in many regions of the domain (particularly in Case 1), shear has a marked influence on the TKE and particularly during the transition towards the nighttime period. To normalize the vertical profiles of variances, fluxes, and TKE budget, we apply the scaling approach proposed by Moeng and Sullivan (1994), which takes into account both buoyancy and shear effects, for the period when the surface heat flux is positive. The velocity scale $w_{m}$ is defined as $w_{m}^{3}=w_{*}^{3}+5 u_{*}^{3}$ while the temperature scale is $\theta_{m}=\overline{w^{\prime} \theta^{\prime}}{ }_{s} / w_{m}$, where $w_{*} \equiv\left[\left(g / T_{0}\right) \overline{w^{\prime} \theta^{\prime}}{ }_{s} z_{i}\right]^{1 / 3}$ is the convective velocity proposed by Deardorff (1972) and $g / T_{0}$ is the buoyancy parameter. This scaling approach assumes a linear combination of convective and surface shear effects and hence neglects all non-linear feedbacks between shear and buoyancy forces. Nevertheless, it has been shown to be a suitable scaling for the secondand third-order moments of turbulence in the sheared convective boundary layer (Moeng and Sullivan 1994). For the moderately stable regime (discussed in the next section), we use $u_{*}$ as the velocity scale since shear is the only mechanism responsible of turbulence production. We also normalize the height $z_{r}$ by the estimate $z_{i}$ shown in Fig. 7.

Here, as an example, we discuss the profiles taken in the middle of the slope shown in Fig. 9. The upper maximum of the normalized $\overline{u^{\prime 2}}$ (at $z_{r} / z_{i} \approx 2$ ), is about 1.8 times larger than the lower value, so the contribution from horizontal advection accounts for a very important part of the turbulence over the slope. The $v$ variance also shows an upper maximum related to advection at the same level. The maximum of the $w$ variance occurs at $z_{r} / z_{i} \approx 0.4$, which is typical of a CBL (Nieuwstadt et al. 1993; Moeng and Sullivan 1994). The profile also shows a secondary maximum at $z_{r} / z_{i} \approx 2.1$, albeit small, which has been observed previously in the sheared CBL (Sorbjan 2004; Conzemius and Fedorovich 2006). The momentum flux $\overline{u^{\prime} w^{\prime}}$ (Fig. 9d) is positive very close to the surface $\left(z_{r} / z_{i}<0.1\right.$ ) due to the upslope westward wind, and negative between $z_{r} / z_{i} \approx 0.1$ and 0.7 due to the strong vertical shear of the induced valley flow. The upper maximum at about $z_{r} / z_{i}=2.2$ may be due to the presence of gravity waves. The shear contribution from $\overline{v^{\prime} w^{\prime}}$ (Fig. 9e) is less important and limited to the levels below $z_{r} / z_{i} \approx 0.5$. The vertical profile of heat flux (Fig. 9f) shows a nearly linear decrease with height, as in a typical convective PBL. However, unlike a typical CBL, there is little negative buoyancy flux at the PBL top, due to the weak capping inversion layer (see Fig. 6) as a result of the interaction with the horizontal breeze and the effect of the return current. The above second-moment profiles also show that the resolved part of the turbulence dominates the subgrid-scale part except at the first few levels close to the ground (below $z_{r} / z_{i} \approx 0.1$ ) where the grid spacing, even with a vertically stretched grid, is still not fine enough to fully resolve the energy-containing eddies.

\subsection{TKE Budget Analysis}

To clarify the nature of the turbulence, the TKE budget is analyzed in this section. The governing equation for the total (resolved plus subgrid) turbulent kinetic energy $E$ is: 

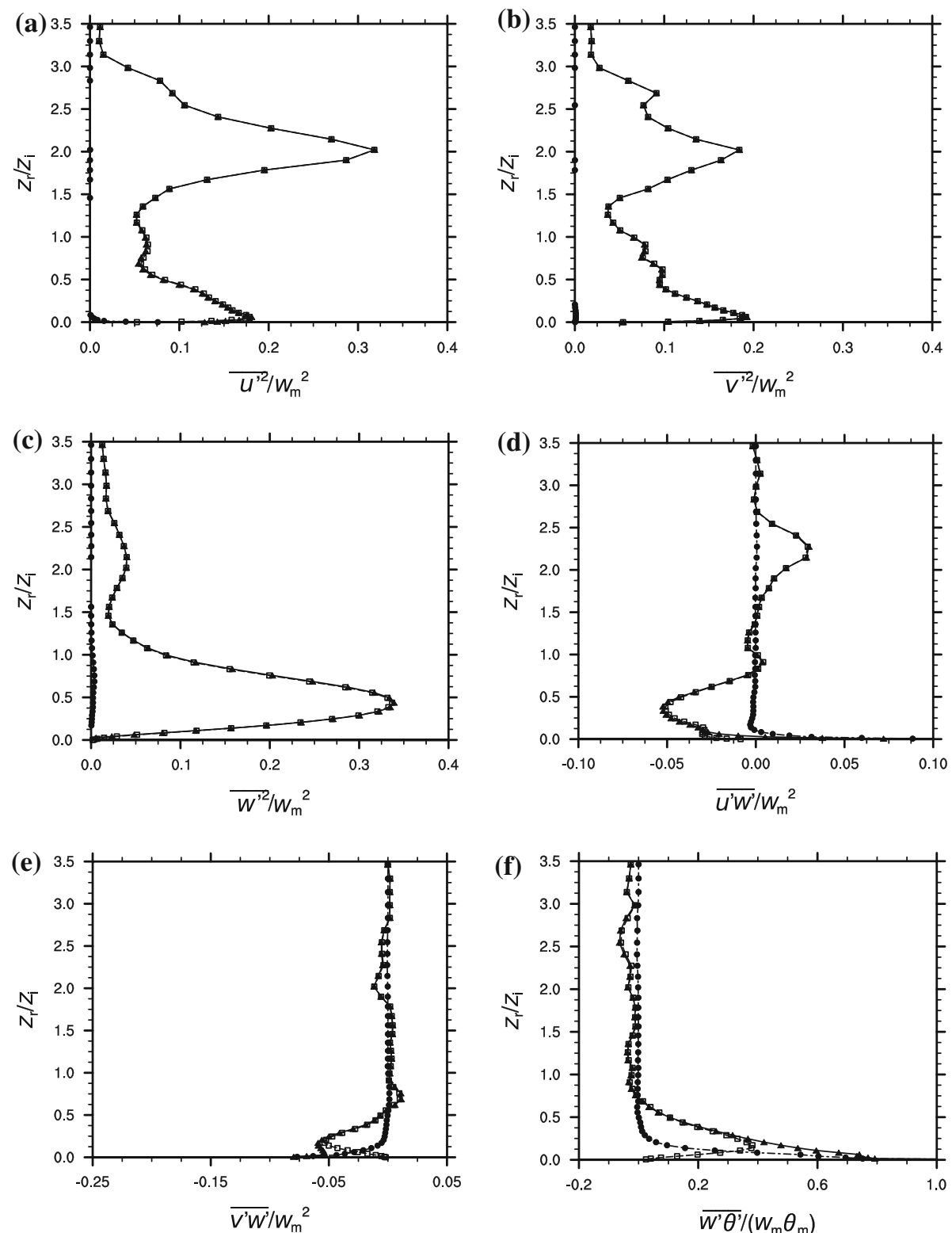

$\rightarrow$ RES+SGS $\quad-\bullet-$ RES $\quad-\cdots$ SGS

Fig. 9 Normalized vertical profiles of the $y$-averaged variances and fluxes: $\overline{u^{\prime 2}} / w_{m}^{2}(\mathbf{a}), \overline{v^{\prime 2}} / w_{m}^{2}(\mathbf{b}), \overline{w^{\prime 2}} / w_{m}^{2}$ (c), $\overline{w^{\prime} \theta^{\prime}} /\left(w_{m} \theta_{m}\right)(\mathbf{d}), \overline{u^{\prime} w^{\prime}} / w_{m}^{2}(\mathbf{e}), \overline{v^{\prime} w^{\prime}} / w_{m}^{2}(\mathbf{f})$ at $x=5,000 \mathrm{~m}, t=7 \mathrm{~h}$ for Case $1\left(V_{g}=2 \mathrm{~m} \mathrm{~s}^{-1}\right)$. Open squares/dashed lines represent the resolved contribution, filled circles/dash-dotted lines shows the subgridscale contribution, filled triangles/full lines represent the total variable 


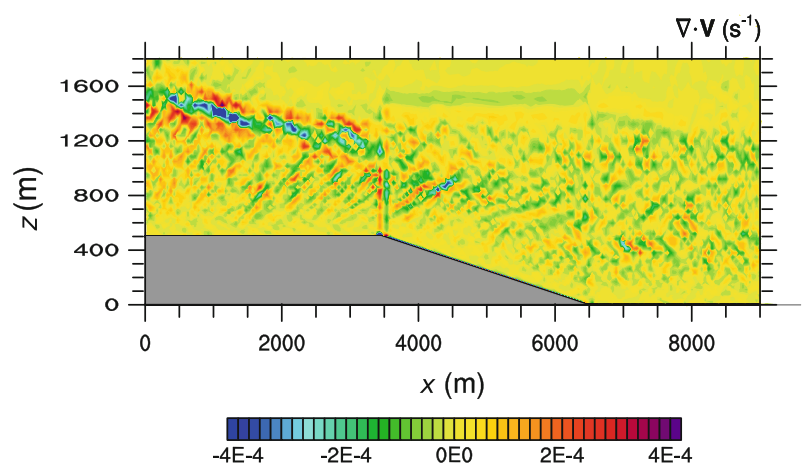

Fig. 10 Vertical cross-section of the $y$-averaged divergence of the wind field at $t=7 \mathrm{~h}$ for Case 1 ( $V_{g}=$ $2 \mathrm{~m} \mathrm{~s}^{-1}$ )

$$
\begin{aligned}
& \frac{\partial E}{\partial t}=-\underbrace{\left(\bar{u} \frac{\partial E}{\partial x}+\bar{w} \frac{\partial E}{\partial z}\right)}_{\mathrm{I}} \\
& -\underbrace{\left(\overline{u^{\prime 2}} \frac{\partial \bar{u}}{\partial x}+\overline{u^{\prime} w^{\prime}} \frac{\partial \bar{w}}{\partial x}+\overline{u^{\prime} v^{\prime}} \frac{\partial \bar{v}}{\partial x}+\overline{w^{\prime 2}} \frac{\partial \bar{w}}{\partial z}+\overline{u^{\prime} w^{\prime}} \frac{\partial \bar{u}}{\partial z}+\overline{v^{\prime} w^{\prime}} \frac{\partial \bar{v}}{\partial z}\right)}_{\text {II }}+\underbrace{\frac{g}{\theta} \overline{w^{\prime} \theta^{\prime}}}_{\text {III }} \\
& -\underbrace{\left\{\frac{\partial}{\partial x}\left[\overline{u^{\prime}\left(E+\frac{p^{\prime}}{\rho}\right)}\right]+\frac{\partial}{\partial z}\left[\overline{w^{\prime}\left(E+\frac{p^{\prime}}{\rho}\right)}\right]\right\}+\overline{\left(E+\frac{p^{\prime}}{\rho}\right)\left(\frac{\partial u^{\prime}}{\partial x}+\frac{\partial v^{\prime}}{\partial y}+\frac{\partial w^{\prime}}{\partial z}\right)}-\varepsilon}_{\text {IV }},
\end{aligned}
$$

where $\rho$ and $p$ are deviations from the background hydrostatic profiles of the density $\rho_{0}$ and pressure $p_{0}$. The terms in I represent the horizontal (ADV_h) and vertical (ADV_v) advection, II contains the horizontal and vertical shear production (S), III the buoyancy production (B), and the last term $\varepsilon$ accounts for small-scale turbulent dissipation. The first two terms in IV represent the horizontal and vertical redistribution of turbulence by the pressure fluctuations (P) and turbulent transport (T), while the last term is the return-to-isotropy contribution, which is zero for an incompressible flow field. In the above equation we have implicitly neglected, for simplicity, the advection and shear generation along $y$. The horizontal shear production (first three terms of II) is negligible in all analyzed locations (not shown), despite the fact that $\overline{u^{\prime} v^{\prime}}$ (Fig. 8h) is large and varying from location to location; this is because $\overline{u^{\prime} v^{\prime}}$ and $\partial v / \partial x$ are poorly correlated.

The WRF model is compressible and the pressure field is computed diagnostically from the equation of state. We checked the flow divergence $\nabla \cdot \mathbf{V}=\partial u / \partial x+\partial v / \partial y+\partial w / \partial z$ and found its magnitude significant (on the order of $10^{-4} \mathrm{~s}^{-1}$ ) even in the shallow PBL. The divergence field is almost uniform in $y$ (not shown). The $y$-averaged flow divergence is shown in Fig. 10; it reveals wavy structures characterized by alternating bands of positive and negative values with about $30^{\circ}$ orientation with respect to the ground. A large band is observed above the plateau, in correspondence with the region characterized by large negative buoyancy (Fig. 8e). The kink at the top of the slope may be an effect of the pressure coordinate due to the sharp change in terrain inclination. With this large flow divergence field, 

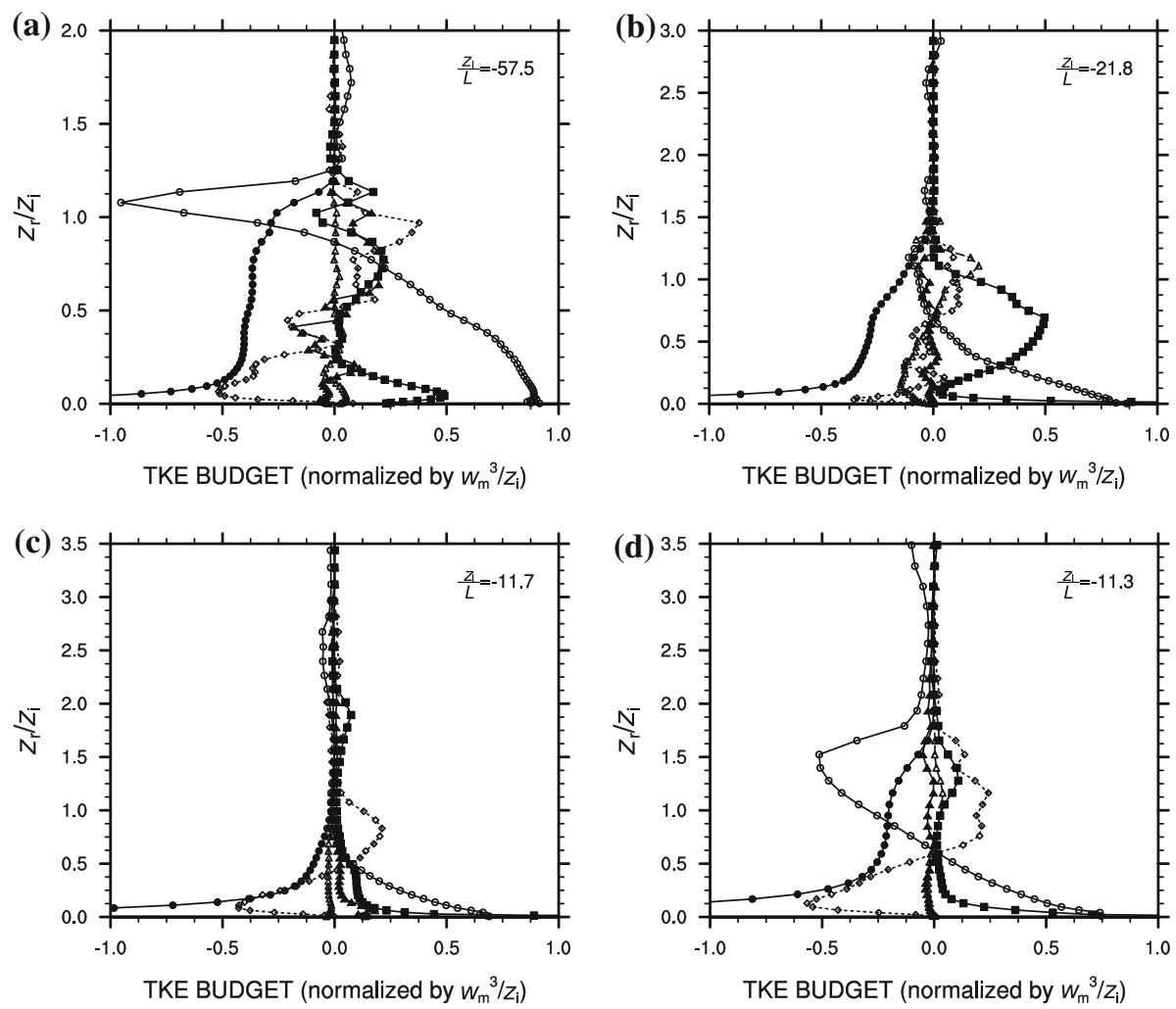

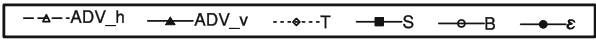

Fig. 11 Normalized vertical profiles of the $y$-averaged TKE budget: $x=250 \mathrm{~m}(\mathbf{a}), x=1,750 \mathrm{~m}(\mathbf{b}), x=$ $5,000 \mathrm{~m}(\mathbf{c}), x=9,000 \mathrm{~m}(\mathbf{d})$ at $t=7 \mathrm{~h}$ for Case $1\left(V_{g}=2 \mathrm{~m} \mathrm{~s}^{-1}\right)$. The curves represent the different terms in (11): $\varepsilon$ (filled circles/full lines) is the dissipation, B (open circles/full lines) is the buoyancy, $\mathrm{S}$ (filled squares/full lines) is the shear production, T (open diamonds/dotted lines) is the turbulent transport, ADV_v (filled triangles/full lines) and ADV_h (open triangles/dashed lines) are, respectively, the vertical and horizontal advection terms. The stability parameter $z_{i} / L$, where $L$ is the Obukhov length, shows the presence of different regimes over the domain

we cannot separate the turbulent pressure fluctuations from those due to flow compressibility. Hence we do not calculate the pressure term P.

To better characterize the nature of turbulence and to intercompare the TKE budget profiles in different zones of the domain, the quantities in Fig. 11 are normalized by $w_{m}^{3} / z_{i}$. Also, to show the relative importance of shear and buoyancy in different stability regimes of the PBL, plots have been labelled with Deardorff's stability parameter $z_{i} / L$, where $L$ is the Obukhov length. The upper boundaries of the vertical axis in Fig. 11a and b reach the top of the domain.

Comparison of the four frames in Fig. 11, which represent four selected locations, reveals significantly different TKE budgets, even though they are all taken at the same time period $(t=7 \mathrm{~h})$. At the west-end boundary $(x=250 \mathrm{~m})$, buoyancy production and small-scale dissipation are the two major terms in the TKE budget (Fig. 11a). This is consistent with the extremely negative stability parameter, $z_{i} / L=-57.5$. However, due to the presence of 
a strong updraft (Fig. 5c), along with a significant negative vertical gradient of TKE in the upper half of the PBL (Fig. 8a), there is also a significant contribution from shear production $\mathrm{S}$, turbulence transport $\mathrm{T}$ and vertical advection. A negative buoyancy flux layer about $300 \mathrm{~m}$ thick is also present between $z_{r} / z_{i} \approx 0.8$ and 1.2 ; such unusual large negative $\mathrm{B}$ has also been reported by Patton et al. (2005) in their large-eddy simulation of a flat terrain with surface heterogeneities. We cannot find any budget term to balance this large negative buoyancy and discuss this later.

At $x=1,750 \mathrm{~m}$ (Fig. 11b), which is still over the ridge but has a strong influence from the valley-flow circulation, the vertical shear production becomes the primary source of TKE between $z_{r} / z_{i} \approx 0.3$ and 1.2, while buoyancy dominates only in the lower part of the PBL. Note that the PBL at this location has $z_{i} / L=-21.8$ and, according to Deardorff (1972), should be dominated by buoyancy production. The horizontal advection is also significant, being negative in the lower half of the PBL but positive in the upper part. The redistribution term $\mathrm{T}$ is quite small, except very close to the surface.

In the middle of the slope at $x=5,000 \mathrm{~m}$ (Fig. 11c), the TKE budget is dominated by buoyancy production and small-scale dissipation in the lower half of the PBL. Near the ground (up to $z_{r} / z_{i} \approx 0.3$ ), positive contributions from vertical shear and vertical advection are also significant. A significant redistribution of energy by turbulent transport from the lower half to the upper part of the PBL is also observed as at the other three locations. This term is likely to be counterbalanced somewhat by the pressure redistribution $\mathrm{P}$ term in the CBL (Moeng and Sullivan 1994) but unfortunately the P term cannot be estimated, as mentioned before. In the middle of the valley (Fig. 11d) both buoyancy and shear are large production terms below $z_{r} / z_{i} \approx 0.5$, although buoyancy still dominates. Above $0.5 z_{i}$, buoyancy becomes a large sink for TKE; the large TKE sink between $z_{r} / z_{i} \approx 0.5$ and 1.7 is only partially balanced by the vertical transport of turbulence and the shear production. Advection plays a negligible role in the TKE budget in the valley. A local maximum of shear production can be observed at $z_{r} / z_{i} \approx 1$.3, which is induced by the vertical shear of mean $v$ as evidenced from Fig. 5b. The stability parameter over the valley is comparable to that over the slope but the TKE budgets in these locations (Fig. 11c, d) are similar only in the lower half of the PBL. The major difference in the TKE budget above $z_{r} / z_{i}=0.5$ in these two locations is the much larger negative buoyancy term associated with the very strong capping inversion over the valley. This unusually large negative buoyancy in the capping inversion is also observed at $x=250 \mathrm{~m}$.

To better clarify the nature of the large negative buoyancy in the inversion layers at $x=250$ and 9,000 m, a quadrant analysis of the vertical heat flux was performed following Sullivan et al. (1998). Figure 12 shows the resolved contribution of the normalized heat flux from the four quadrants: $\overline{w^{+} \theta^{+}}, \overline{w^{+} \theta^{-}}, \overline{w^{-} \theta^{-}}, \overline{w^{-} \theta^{+}}$, where + and - represent the positive and negative fluctuations from the $y$ averages, as well as the total fluxes. In both locations wave activity is present in the capping zone and above as suggested in Fig. 10. In the lower half of the PBL, the total flux is dominated by the two positive quadrants, particularly by $\overline{w^{+} \theta^{+}}$at $x=250 \mathrm{~m}$ (Fig. 12a) due to the strong updrafts.

In the inversion layer at both locations, the two positive quadrants have similar magnitudes, as do the two negative quadrants, which suggest the presence of wave motions. However, the magnitudes of the two negative quadrants are much larger than those of the two positive quadrants, which results in the very negative buoyancy flux there. Our quadrant distributions in the inversion layer also differ from those of the entrainment heat flux analyzed by Sullivan et al. (1998) where the entrainment flux is dominated by the second (cold air rising) and the third (cold air sinking) quadrants. This suggests that our negative heat flux is not due to entrainment. This puzzling negative heat flux in the inversion layer results in an 

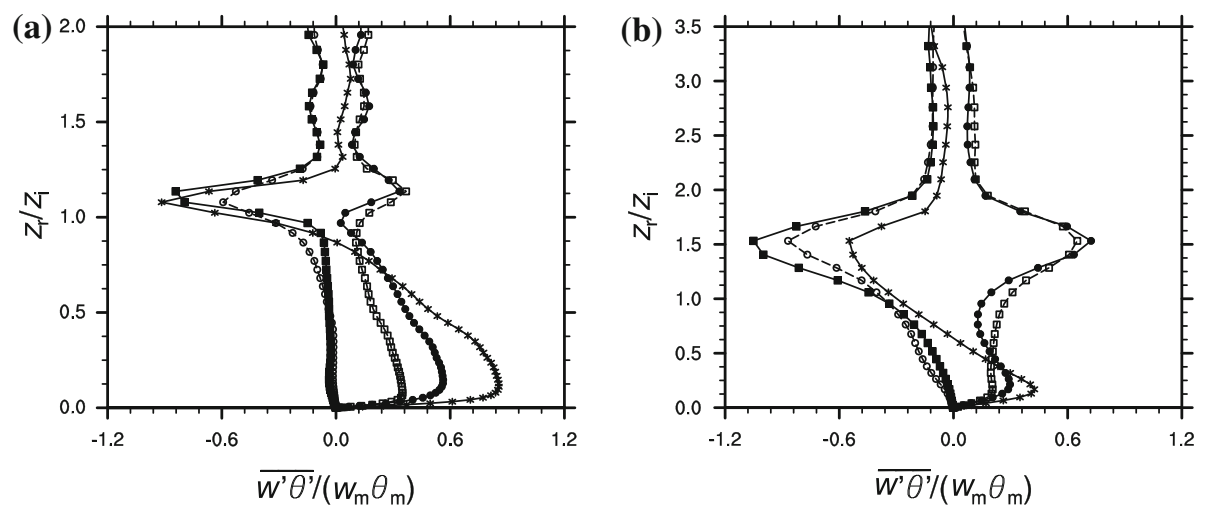

Fig. 12 Normalized vertical profiles of the $y$-averaged resolved buoyancy flux partitioned into four quadrants: $x=250 \mathrm{~m}(\mathbf{a}), x=9,000 \mathrm{~m}(\mathbf{b})$ at $t=7 \mathrm{~h}$ for Case $1\left(V_{g}=2 \mathrm{~m} \mathrm{~s}^{-1}\right)$. The signs + and - stand for the positive and negative fluctuations with respect to the $y$ averages. The total resolved heat-flux is shown by the asterisk/full lines

unbalanced TKE budget that may be related to the compressibility of the WRF model; as shown in Fig. 10, the flow divergence is non-zero and hence the role of pressure in redistributing energy in the simulated turbulent flow is questionable. As will be shown in the next section, this large negative buoyancy and the associated imbalance in the TKE budget is not observed in the presence of a stronger along-valley flow. This can be attributed to a damping of the waves associated to the pressure fluctuations as the shear production terms increase. Further investigation is needed.

\section{Influence of a Strong Geostrophic Wind Along the Valley}

We now examine Case 2, which has a larger geostrophic wind $V_{g}=10 \mathrm{~m} \mathrm{~s}^{-1}$ compared to Case 1; all other parameters of the two simulations remain the same. Our discussion below focuses mainly on the differences between the two cases to signify the effect of geostrophic forcing. The maximum values of the surface heat flux are roughly the same as those in Case 1 but appear $1 \mathrm{~h}$ earlier (not shown). Again, at $t=9.5 \mathrm{~h}$ the positive surface heat flux becomes zero or slightly negative throughout the domain; however, the sign change occurs earlier (at about $6.5 \mathrm{~h}$ ) over the valley. So, at $t=7 \mathrm{~h}$ we have an unstable PBL over the ridges and the slopes, but a weakly stable PBL over the valley. As in Case 1, the most negative heat flux occurs over the slopes at $t=11 \mathrm{~h}$, but with a much larger magnitude; this time period coincides with the onset of downslope winds (not shown). The differences between the two cases can be explained by the feedbacks between shear and buoyancy forces. The kinematic surface heat flux is given by:

$$
\overline{w^{\prime} \theta^{\prime}}{ }_{s}=k u_{*}\left(\theta_{s}-\theta_{1}\right) \frac{1}{\varphi_{h}}
$$

where $k$ is the Von Karman constant, $\theta_{1}$ is the temperature at the first model layer and $\varphi_{h}$ is a stability function. Initially, surface shear generated by both the valley circulation and the geostrophic wind contributes to enhance $u_{*}$ and thus $\overline{w^{\prime} \theta^{\prime}}{ }_{s}$. Later, when the mixed layer 
grows, the shear generated by the geostrophic wind is reduced (because $\partial v / \partial z$ becomes smaller). The shear generated by the baroclinic circulation is instead sustained by the surface temperature increase. This explains why the maximum values of the surface heat flux are reached earlier in Case 2 but are roughly the same for both cases. The transport of warm air from the upper levels towards the ground by subsidence in the valley causes the decrease of $\left(\theta_{s}-\theta_{1}\right)$ and hence of the surface heat flux. In addition, as shown later, the capping inversion over the valley is much stronger in Case 1, which effectively isolates the warm inversion air from reaching to the first grid level. Thus, over the valley the sign change of $\left(\theta_{s}-\theta_{1}\right)$ occurs later in Case 1 . In other regions of the domain, the sign change of the surface heat flux occurs at the same time in both simulations. When the surface heat flux becomes negative, its magnitude is increased by the wind shear, and a negative value of $\overline{w^{\prime} \theta^{\prime}}{ }_{s}$ drives the onset of downslope winds over the slopes. The other shear source in moderately stable conditions is the geostrophically forced wind shear, and therefore the higher negative values are observed in Case 2 over the slopes. Figure 13 shows the distribution of mean winds and potential temperature for Case 2. The thermally-driven mean circulation is similar to that in Case 1, but with a stronger upslope wind, a weaker return current and a weaker horizontal breeze (Fig. 13a). Also, its return current is deeper, extending up to $1,700 \mathrm{~m}$, while the depth of the upslope wind remains equal to that of Case 1. The mean $v$ wind (Fig. 13b) shows no tunnelling over the valley due to the lack of a capping inversion layer (Fig. 13d). The mean vertical velocity (Fig. 13c) reveals a stronger updraft over the west end of the ridge than that in Case 1, and also shows several updrafts along the slopes and in the valley. These updrafts are associated with streaks along the $y$-direction over the foot of the slope and the valley, which extend vertically through the entire PBL depth (not shown). Of particular evidence is a counter-clockwise vortex street along the foot of the slope, which extends horizontally from $x \approx 6,200 \mathrm{~m}$ to $x \approx 7,500 \mathrm{~m}$.

On average $z_{i}$ is characterized by a more rapid evolution and the PBL top reaches higher levels compared to Case 1, in agreement with the observations taken for various strongly sheared convective boundary layers by Fedorovich and Conzemius (2008). The maximum value of $z_{i} \approx 950 \mathrm{~m}$ (vs. $\approx 850 \mathrm{~m}$ in Case 1 ) is attained at $t=7 \mathrm{~h}$ near the side boundaries over the ridges and persists until $9 \mathrm{~h}$; after that time a sharp decrease in the PBL height accompanies the vanishing of the positive surface heat flux over the ridges (not shown). There is also a deepening of the PBL in proximity of the base of the slopes during the transition to the weakly stable regime occurring from 7 to $9 \mathrm{~h}$; here the depth of about $600 \mathrm{~m}$ is associated with the formation of two-dimensional rolls, as evidenced from the mean wind fields (Fig. 13a, c).

Maximum TKE values are about 1.7 times higher than those in Case 1 (not shown), confirming the noticeable influence of the wind shear, especially in the upper part of the PBL. As in Case 1, the maximum TKE occurs near the west-end boundary. A fairly good correlation between $z_{i}$ and the level at which TKE decreases significantly is observed only over the slope and the eastern part of the ridge, where the PBL top again reveals an angle close to the slope inclination.

Next we apply the scaling described in Sect. 3.5 to the vertical profiles of second-moment statistics, again taken in the middle of the slope (where the surface heat flux is still positive). The lower maximum of the $u$ variance (Fig. 14a) is slightly greater than that in Case 1 . The secondary maximum in Case 1 above the PBL disappears here, which will be shown later to relate to the larger geostrophic wind that reduces the horizontal advection along $x$. The $v$ variance profile (Fig. 14b) is similar to Case 1, although the surface maximum is 1.5 times larger and the upper peak is shifted upward to $z_{r} / z_{i} \approx 3$; this shift can be explained by the presence of intense wind shear at this level. As expected for a less buoyancy dominant 

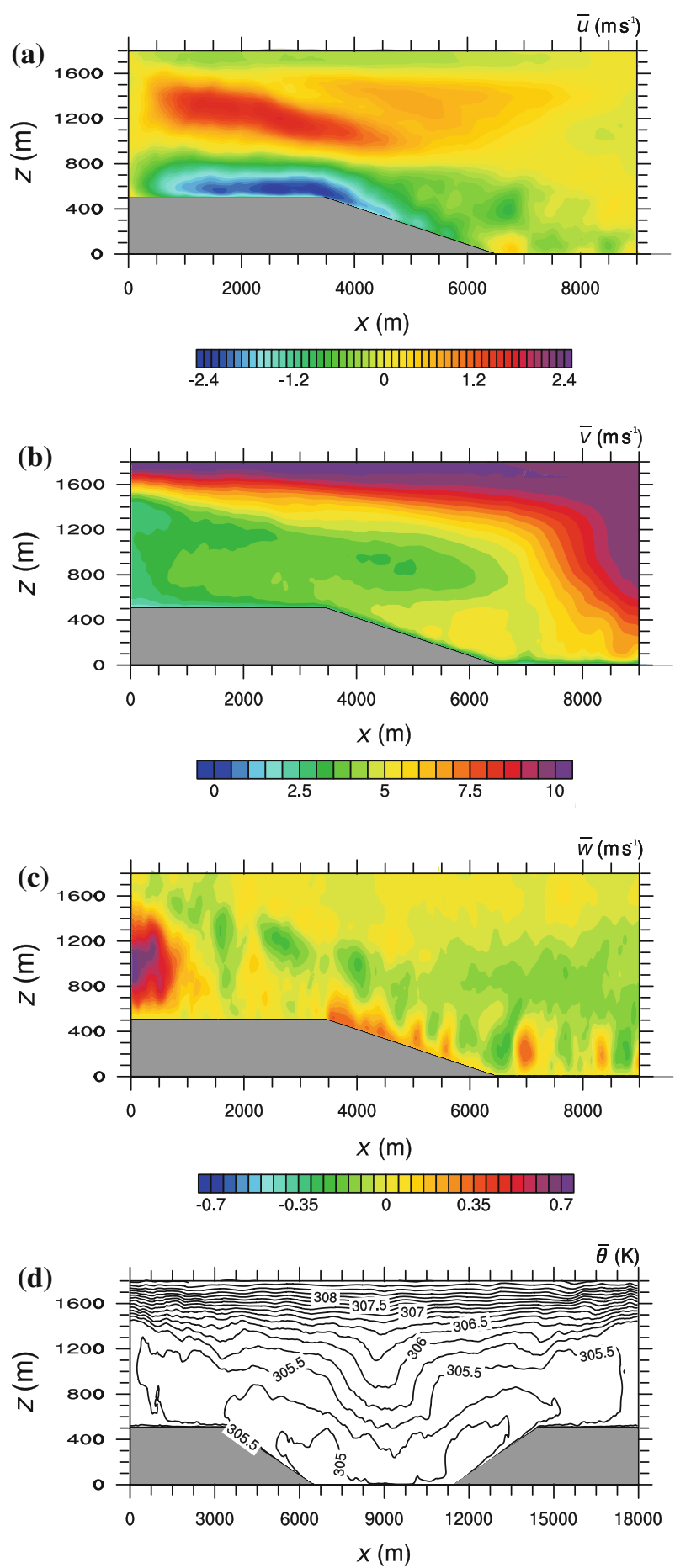

Fig. $13 y$-averaged vertical cross-sections of the wind components: $\bar{u}(\mathbf{a}), \bar{v}(\mathbf{b}), \bar{w}(\mathbf{c})$ and isotherms (d) at $t=7 \mathrm{~h}$ for Case $2\left(V_{g}=10 \mathrm{~m} \mathrm{~s}^{-1}\right)$ 

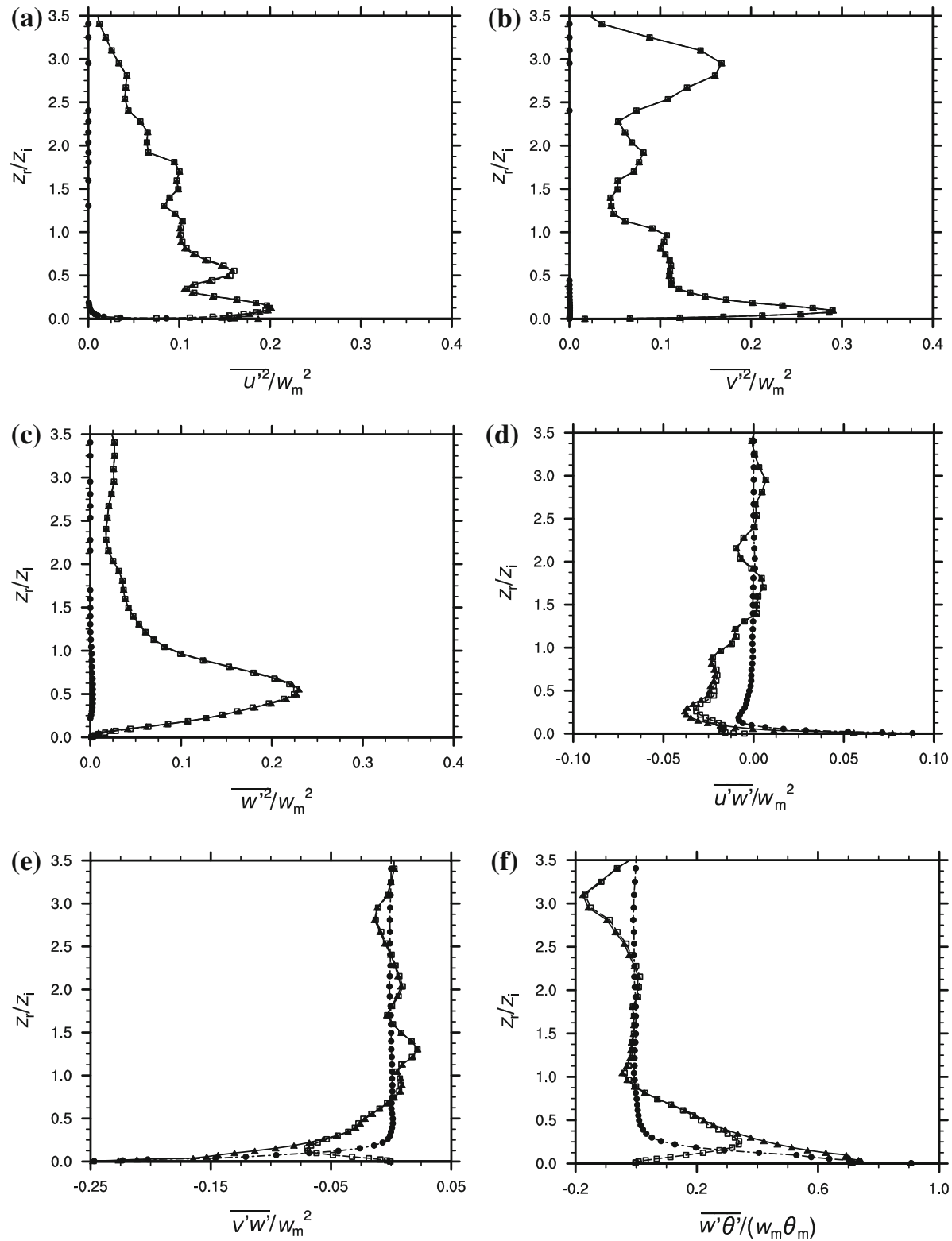

$\leadsto$ RES+SGS $\quad$ - - RES $-\bullet-$ SGS

Fig. 14 Normalized vertical profiles of the $y$-averaged variances and fluxes: $\overline{u^{\prime 2}} / w_{m}^{2}$ (a), $\overline{v^{\prime 2}} / w_{m}^{2}$ (b), $\overline{w^{\prime 2}} / w_{m}^{2}$ (c), $\overline{w^{\prime} \theta^{\prime}} /\left(w_{m} \theta_{m}\right)(\mathbf{d}), \overline{u^{\prime} w^{\prime}} / w_{m}^{2}(\mathbf{e}), \overline{v^{\prime} w^{\prime}} / w_{m}^{2}$ (f) at $x=5,000 \mathrm{~m}, t=7 \mathrm{~h}$ for Case 2 $\left(V_{g}=10 \mathrm{~m} \mathrm{~s}^{-1}\right)$. Open squares/dashed lines represent the resolved contribution, filled circles/dash-dotted lines shows the subgrid-scale contribution, filled triangles/full lines represent the total variable

PBL, the normalized vertical velocity variance (Fig. 14c) peak is less than that of Case 1 and located higher at $z_{r} / z_{i} \approx 0.5$, which agrees with the LES results of Sorbjan (2004) for a strongly sheared baroclinic convective boundary layer. The normalized $\overline{u^{\prime} w^{\prime}}$ profile 

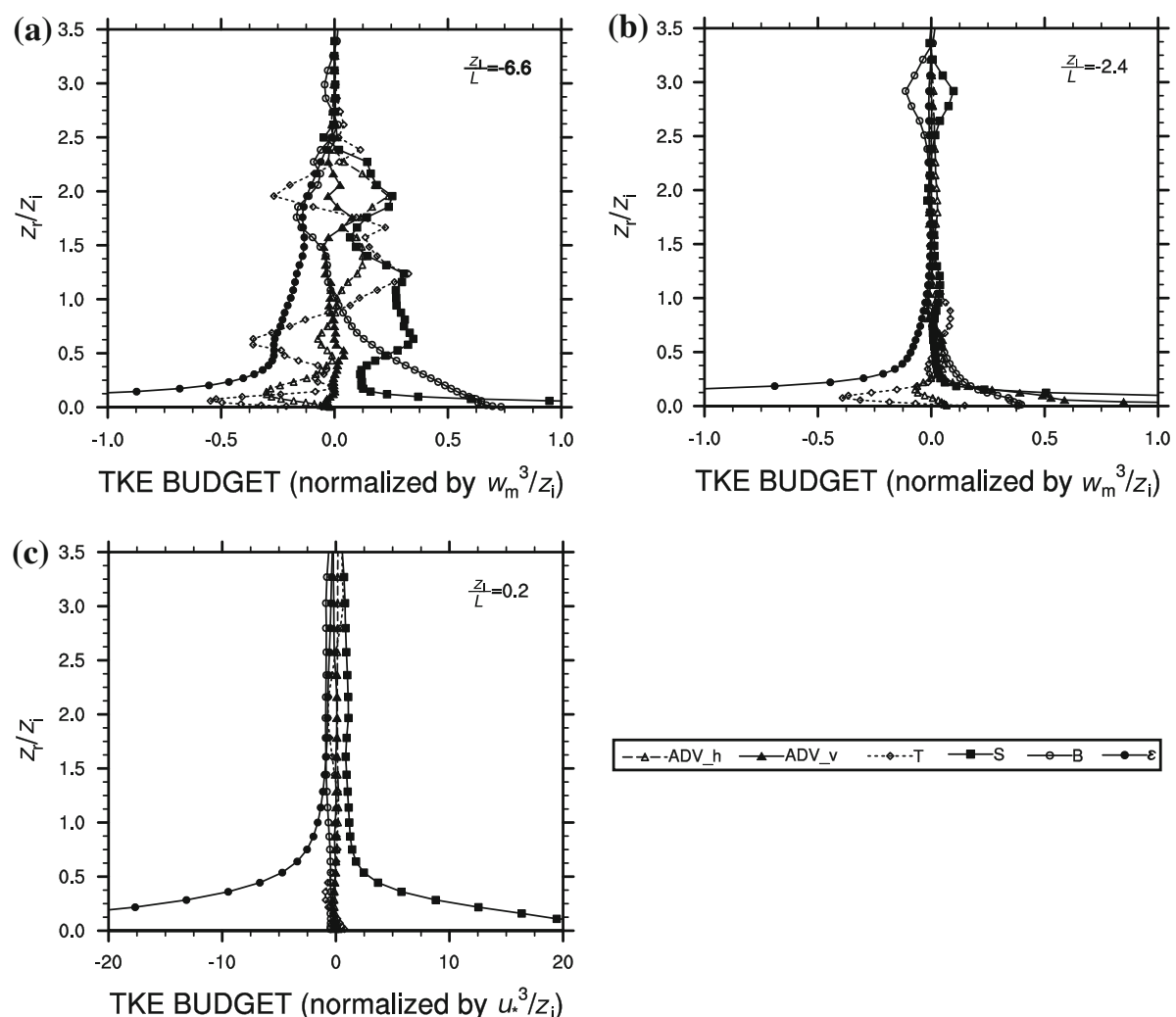

Fig. 15 Normalized vertical profiles of the $y$-averaged TKE budget: $x=1,750 \mathrm{~m}(\mathbf{a}), x=5,000 \mathrm{~m}(\mathbf{b}), x=$ $9,000 \mathrm{~m}(\mathbf{c})$ at $t=7 \mathrm{~h}$ for Case $2\left(V_{g}=10 \mathrm{~m} \mathrm{~s}^{-1}\right)$. The curves represent the different terms in (11): $\varepsilon$ (filled circles/full lines) is the dissipation, B (open circles/full lines) is the buoyancy, S (filled squares/full lines) is the shear production, T (open diamonds/dotted lines) is the turbulent transport, ADV_v (filled triangles/full lines) and ADV_h (open triangles/dashed lines) are, respectively, the vertical and horizontal advection terms. The stability parameter $z_{i} / L$, where $L$ is the Obukhov length, shows the presence of different regimes over the domain

(Fig. 14d) is similar to that of Case 1, but the negative value is smaller and extends up to the top of the PBL; also, the secondary peak in Case 1 at $z_{r} / z_{i} \approx 2.2$ is not present here. Vigorous surface shear is shown in the $\overline{v^{\prime} w^{\prime}}$ profile (Fig. 14e) up to $0.5 z_{i}$; the magnitudes are about 2.5 times higher than those in Case 1. A modest positive flux is shown just above the PBL top, which may be associated with the presence of gravity waves. It is interesting to note that, in this case such an upper-level secondary large flux appears in the $v$-flux profile, while in the case of the weak geostrophic condition, the secondary large flux shows up in the $u$ flux. The heat flux (Fig. 14f) is similar to that in Case 1 except for the presence of a deep negative flux layer between $z_{r} / z_{i} \approx 2.5$ and 3.5; as shown later, this is related to the strong shear generation of turbulence at this level.

The normalized TKE budget at various locations is shown in Fig. 15, and again differs significantly from location to location. In the middle of the ridge (Fig. 15a) the buoyancy production is important in the lower half of the domain, while the vertical shear dominates in both the surface layer and the upper half of the PBL. An entrainment layer (i.e., the negative buoyancy flux layer) is present between $z_{r} / z_{i} \approx 1.5$ and 2 . Horizontal advection is negative 
in the lower half of the PBL and becomes positive above $z_{r} \approx z_{i}$, and between $z_{r} / z_{i} \approx 1$ and 2 both horizontal advection and vertical shear contribute significantly to the production of TKE, and which balance dissipation and the negative buoyancy. The stability parameter $z_{i} / L=-6.6$ indicates an unstable regime, but its magnitude is about 3 times smaller than that in Case 1 due to the increasing importance of surface shear. The redistribution by turbulent transport is negative below $z_{r} / z_{i} \approx 0.8$ and positive up to $z_{r} / z_{i} \approx 2$.

In the middle of the slope (Fig. 15b) the most important contributions to the production of turbulence are the shear and the vertical advection up to $z_{r} / z_{i} \approx 0.2$, while in the remaining part of the PBL, buoyancy prevails. At $z_{r} / z_{i} \approx 3$, in correspondence with the interface between the return current and the stratified atmosphere above, buoyancy destruction in the entrainment zone is nearly balanced by the shear. In the middle of the valley (Fig. 15c) we have near-neutral conditions $\left(z_{i} / L=0.2\right)$ and hence the friction velocity is the appropriate parameter for scaling the TKE budget profiles. The small-scale dissipation nearly balances the shear production, which is typical of a near-neutral PBL (e.g., Moeng and Sullivan 1994).

It is worth noting that Case 2 does not show any imbalance in the TKE budget at any location, and can be attributed to a damping of the wave modes excited by convection (and the resulting pressure correlations) in the presence of a greater shear generation.

\section{Summary and Conclusions}

The turbulent structure of a complex PBL driven by (a) a non-uniform surface heating, and (b) an along-valley geostrophic wind $V_{g}$ is investigated by means of large-eddy simulation (LES). The three-dimensional non-hydrostatic meteorological model WRF is modified to include a new formulation for the SGS length scale. Turbulent flow over a ridge-valley topography, symmetrical in $x$ and uniform in $y$, is simulated with a LES grid resolution to explicitly resolve the energy-containing turbulent eddies. The thermally driven circulation is generated by imposing a sinusoidal time change of the surface temperature; the surface heat and momentum fluxes are then computed based on Monin-Obukhov surface similarity theory. The approach results in a simulated surface heat flux that responds to the valley-flow circulation. We focus on the daytime circulation, i.e., the upslope flow. Two cases are simulated, with $V_{g}=2$ and $10 \mathrm{~m} \mathrm{~s}^{-1}$, to investigate the influence of the along-valley geostrophic wind on turbulence properties.

Turbulence statistics vary from location to location due to topography, and we define the ensemble statistics as averages along $y$ (along the valley) and also over a time period of $40 \mathrm{~min}$. The first-moment statistics show the characteristics of the mean flow, which consists of two symmetrical upslope circulations. The surface heat flux reveals complicated time and space distributions. The largest positive surface flux occurs about $2 \mathrm{~h}$ before the maximum ground temperature and during the transition from upslope to downslope flows the largest negative flux occurs near the foot of the slope. In both cases the PBL over the ridge is much deeper than that in the valley, and the growth line of the PBL over the slope (and even extending into the ridge) roughly corresponds to the slope angle. The PBL structure differs from the idealized CBL over a uniform surface. Even when the stability parameter indicates a very unstable CBL, the shear production is as important as (or even dominates) the buoyancy production in generating turbulence in many regions, particularly over the ridge where the valley-flow circulation creates a strong vertical shear of the mean $u$. The TKE budget varies significantly at different regions over ridge, slope and valley.

For the case $V_{g}=2 \mathrm{~m} \mathrm{~s}^{-1}$, a horizontal breeze, from valley towards the ridge, appears near the top of the PBL (which happens to be at about the ridge height) over the valley and extends 
to the slope region. This breeze merges with the upslope wind and significantly increases its depth at the top of the slope. The horizontal breeze, along with the return current, also leads to a secondary circulation over the valley, which may significantly affect the vertical distribution of air pollution in the valley. Associated with this westward horizontal breeze is a strong northward wind (tunnelling along the valley) again at about the ridge height. For the case $V_{g}=10 \mathrm{~m} \mathrm{~s}^{-1}$, the horizontal breeze is weaker and the tunnelling wind disappears, which may be due to the change in strength and height of the capping inversion over the valley.

For the case $V_{g}=2 \mathrm{~m} \mathrm{~s}^{-1}$, a strong capping inversion forms over the valley at a height of about $400 \mathrm{~m}$, which is about the ridge height, as a result of the strong subsidence induced by the return current of the thermal circulation. This capping inversion weakens for the case $V_{g}=10 \mathrm{~m} \mathrm{~s}^{-1}$ because of: (a) a deeper earlier CBL over the valley, which grows above the ridge height, and (b) the formation of a two-dimensional roll-like structure over the valley and at the foot of the slope due to the larger shear during the transition to the stable regime. The TKE over the slope exhibits a secondary peak above the PBL due to the horizontal advection of turbulence (carried by the return current), which may significantly affect the dispersion process there. With strong geostrophic wind forcing, this horizontal advection effect is much reduced, but a large $v$ variance appears at $z_{r} \approx 3 z_{i}$ over the slope due to the much stronger shear at that level. Over the slope, the CBL reveals some unusual features. For example, in both cases, the capping inversion at the top of the CBL is largely diminished due to the formation of the horizontal breeze and the return current. For the case $V_{g}=10 \mathrm{~m} \mathrm{~s}^{-1}$, an entrainment heat flux occurs at the interface between the return current and the stratified atmosphere above, around $z_{r} \approx 3 z_{i}$, where the negative buoyancy balances the shear production of TKE.

We show that the LES technique can be used to study higher-moment turbulence statistics such as variances, fluxes, TKE budget, and how they are distributed over different regions of an idealized complex terrain. Despite the fact that these higher moment statistics are of great importance to PBL applications such as air pollution and wind farm siting, they have not been thoroughly investigated at a spatial resolution as high as $50 \mathrm{~m}$ in previous LES studies over variable orography. The results of our study show that, even with a certain degree of idealization, spatial inhomogeneities in terrain elevation considerably modify the mechanisms of turbulence generation and redistribution under convective conditions, introducing significant shear production and advection terms in the TKE budget.

Imposing the surface temperature anomaly as the thermal forcing, even neglecting the interaction with the soil capacity, allows coupling between surface thermal and momentum fluxes, which is a step towards the reproduction of real conditions where the surface heat flux over the slopes significantly differs from that over the valley or the ridges. In fact, most previous LES studies considered a constant stationary surface heat flux, which is not realistic under variable terrain elevation.

The present non-stationary LES investigation reveals that the PBL characteristics (e.g. its depth and the stability regime) are governed by mechanisms that change during the daytime evolution, depending on the relative importance of thermal and shear forcing. This implies that different scaling parameters should be used in the analysis of turbulence statistics at different times of the day and also for different regions of the domain, according to the local stability regime defined by the sign of $z_{i} / L$. Furthermore, the PBL height itself must be defined locally and its determination method depends on the sign of the surface heat flux. Turbulent motions in a valley circulation are not explicitly reproduced by mesoscale models with typical horizontal grids larger than $1 \mathrm{~km}$. Furthermore, PBL schemes currently used in mesoscale models are one-dimensional in height and do not include the effects of horizontal 
transport. We show here that the complex turbulence structure over a valley and its significant horizontal heterogeneity has a large impact on the mesoscale circulation, particularly the buoyancy flux redistribution. This effect should be included as additional source/sink terms in future PBL schemes.

Acknowledgements We thank Richard Rotunno, Edward Patton, Peter Sullivan and Jimy Dudhia for their helpful discussions. The computing resources were provided by NCAR's Mesoscale and Microscale Meteorology (MMM) division. The computations were performed on NCAR's IBM Power 575 "Bluefire" supercomputer. The first author express his gratitude for the full support provided to him by NCAR's MMM division during his short term visit in autumn 2008. NCAR is sponsored by the National Science Foundation, USA.

\section{References}

Antonelli M, Rotunno R (2007) Large-eddy simulation of the onset of the sea breeze. J Atmos Sci 64:44454457

Beare RJ, MacVean MK, Holtslag AAM, Cuxart J, Esau I, Golaz J-C, Jimenez MA, Khairoutdinov M, Kosovic B, Lewellen D, Lund TS, Lundquist JK, McCabe A, Moene AF, Noh Y, Raasch S, Sullivan PP (2006) An intercomparison of large-eddy simulations of the stable boundary layer. Boundary-Layer Meteorol 118:247-272

Brehm M, Freytag C (1982) Erosion of the night-time thermal circulation in an alpine valley. Arch Meteorol Geophys Bioklimatol 31:331-352

Chow FK, Weigel AP, Street RL, Rotach MW, Xue M (2006) High-resolution large-eddy simulations of flow in a steep alpine valley. Part I: Methodology, verification and sensitivity experiments. J Appl Meteorol Climatol 45:63-86

Conzemius RJ, Fedorovich E (2006) Dynamics of sheared convective boundary layer entrainment. Part I: Methodological background and large eddy simulations. J Atmos Sci 63:1151-1178

Deardorff JW (1970) A numerical study of three-dimensional turbulent channel flow at large Reynolds numbers. J Fluid Mech 41:452-480

Deardorff JW (1972) Numerical investigation of neutral and unstable planetary boundary layers. J Atmos Sci 29:91-115

Deardorff JW (1980) Stratocumulus-capped mixed layer derived from a three-dimensional model. BoundaryLayer Meteorol 18:495-527

Fedorovich E, Conzemius R (2008) Effects of wind shear on the atmospheric convective boundary layer structure and evolution. Acta Geophys 56:114-141

Haiden T, Whiteman CD (2005) Katabatic flow mechanisms on a low-angle slope. J Appl Meteorol 44: $113-126$

Hennemuth B (1987) Heating of a small alpine valley. Meteorol Atmos Phys 36:287-296

Hunt JCR, Fernando HJS, Princevac M (2003) Unsteady thermally driven flows on gentle slopes. J Atmos Sci 60:2169-2182

Hyun Y-K, Kim K-E, Ha K-J (2005) A comparison of methods to estimate the height of stable boundary layer over a temperate grassland. Agric For Meteorol 132:132-142

Kondo J, Kuwagata T, Haginoya S (1989) Heat budget analysis of nocturnal cooling and daytime heating in a basin. J Atmos Sci 46:2917-2933

Lundquist KA, Chow FK, Lundquist JK (2010) An immersed boundary method for the weather research and forecasting model. Mon Weather Rev 138:796-817

Manins PC, Sawford BL (1979) A model of katabatic winds. J Atmos Sci 36:619-630

Michioka T, Chow FK (2008) High-resolution large-eddy simulations of scalar transport in atmospheric boundary layer flow over complex terrain. J Appl Meteorol Climatol 47:3150-3169

Moeng C-H (1984) A large-eddy-simulation model for the study of planetary boundary-layer turbulence. J Atmos Sci 41:2052-2062

Moeng C-H, Sullivan PP (1994) A comparison of shear- and buoyancy-driven planetary boundary layer flows. J Atmos Sci 51:999-1022

Moeng C-H, Dudhia J, Klemp J, Sullivan P (2007) Examining two-way grid nesting for large eddy simulation of the PBL using the WRF model. Mon Weather Rev 135:2295-2311

Monti P, Fernando HJS, Princevac M, Chan WC, Kowalewski TA, Pardyjak ER (2002) Observations of flow and turbulence in the nocturnal boundary layer over a slope. J Atmos Sci 59:2513-2534 
Nieuwstadt FTM, Mason PJ, Moeng C-H, Schumann U (1993) Large-eddy simulation of the convective boundary layer: a comparison of four computer codes. In: Durst F et al (eds) Turbulent shear flows, vol 8. Springer, Berlin, $431 \mathrm{pp}$

Noppel H, Fiedler F (2002) Mesoscale heat transport over complex terrain by slope winds-a conceptual model and numerical simulations. Boundary-Layer Meteorol 104:73-97

Patton EG, Sullivan PP, Moeng C-H (2005) The influence of idealized heterogeneity on wet and dry planetary boundary layers coupled to the land surface. J Atmos Sci 62:2078-2097

Princevac M, Fernando HJS (2007) A criterion for the generation of turbulent anabatic flows. Phys Fluids 19:105102. doi:10.1063/1.2775932

Rampanelli G, Zardi D, Rotunno R (2004) Mechanisms of up-valley winds. J Atmos Sci 61:3097-3111

Rotach MW et al (2004) Turbulence structure and exchange processes in an alpine valley: the Riviera project. Bull Am Meteorol Soc 85:1367-1385

Sagaut P (2006) Large-eddy simulation for incompressible flows. Springer, New York, $556 \mathrm{pp}$

Saiki EM, Moeng C-H, Sullivan PP (2000) Large-eddy simulation of the stably stratified planetary boundary layer. Boundary-Layer Meteorol 95:1-30

Schumann U (1990) Large-eddy simulation of the up-slope boundary layer. Q J R Meteorol Soc 116:637-670

Scotti A, Meneveau C, Lilly DK (1993) Generalized Smagorinsky model for anisotropic grids. Phys Fluids 5:2306-2308

Simpson JE (1994) Sea breeze and local winds. Cambridge University Press, Cambridge, $228 \mathrm{pp}$

Skamarock WC, Klemp JB, Dudhia J, Gill DO, Barker DM, Duda MG, Huang X-Y, Wang W, Powers JG (2008) A description of the advanced research WRF version 3. NCAR/TN-475, $113 \mathrm{pp}$

Skyllingstad ED (2003) Large-eddy simulation of katabatic flows. Boundary-Layer Meteorol 106:217-243

Sorbjan Z (2004) Large-eddy simulation of the baroclinic mixed layer. Boundary-Layer Meteorol 112:57-80

Sullivan PP, Mc Williams JC, Moeng C-H (1994) A subgrid-scale model for large-eddy simulation of planetary boundary-layer flows. Boundary-Layer Meteorol 71:247-276

Sullivan PP, Moeng C-H, Stevens B, Lenschow D, Mayor SD (1998) Structure of the entrainment zone capping the convective atmospheric boundary layer. J Atmos Sci 55:3042-3064

Weigel AP, Chow FK, Rotach MW, Street RL, Xue M (2006) High-resolution large-eddy simulations of flow in a steep alpine valley. Part II: Flow structure and heat budgets. J Appl Meteorol Climatol 45:87-107

Wyngaard JC (1983) Lectures on the planetary boundary layer. In: Lilly DK, Gal-Chen T (eds) Mesoscale meteorology-theories, observations and models. D. Reidel Publishing Company, Dordrecht, $781 \mathrm{pp}$ 